\title{
Multiple chemodynamic stellar populations of the Ursa Minor dwarf spheroidal galaxy
}

\author{
Andrew B. Pace ${ }^{\odot},{ }^{1,2,3 \star}$ Manoj Kaplinghat, ${ }^{3}$ Evan Kirby, ${ }^{4}$ Joshua D. Simon, ${ }^{5}$ \\ Erik Tollerud, ${ }^{6}$ Ricardo R. Muñoz, ${ }^{7}$ Patrick Côté, ${ }^{8}$ S. G. Djorgovski ${ }^{4}$ and Marla Geha ${ }^{9}$ \\ ${ }^{1}$ McWilliams Center for Cosmology, Carnegie Mellon University, 5000 Forbes Ave, Pittsburgh, PA 15213, USA \\ ${ }^{2}$ George P. and Cynthia Woods Mitchell Institute for Fundamental Physics and Astronomy, Department of Physics and Astronomy, Texas A\&M University, \\ College Station, TX 77843, USA \\ ${ }^{3}$ Center for Cosmology, Department of Physics and Astronomy, University of California, Irvine, CA 92697, USA \\ ${ }^{4}$ California Institute of Technology, 1200 E. California Blvd., MC 249-17, Pasadena, CA 91125, USA \\ ${ }^{5}$ Observatories of the Carnegie Institution for Science, 813 Santa Barbara Street, Pasadena, CA 91101, USA \\ ${ }^{6}$ Space Telescope Science Institute, 3700 San Martin Drive, Baltimore, MD 21218, USA \\ ${ }^{7}$ Departamento de Astronomía, Universidad de Chile, Camino del Observatorio 1515, Las Condes, Santiago, Chile \\ ${ }^{8}$ Herzberg Astronomy and Astrophysics Research Centre, National Research Council of Canada, 5071 W. Saanich Road, Victoria, BC V9E 2E7, Canada \\ ${ }^{9}$ Astronomy Department, Yale University, New Haven, CT 06520, USA
}

Accepted 2020 May 18. Received 2020 May 11; in original form 2020 February 21

\begin{abstract}
We present a Bayesian method to identify multiple (chemodynamic) stellar populations in dwarf spheroidal galaxies (dSphs) using velocity, metallicity, and positional stellar data without the assumption of spherical symmetry. We apply this method to a new Keck/Deep Imaging Multi-Object Spectrograph (DEIMOS) spectroscopic survey of the Ursa Minor (UMi) dSph. We identify 892 likely members, making this the largest UMi sample with line-of-sight velocity and metallicity measurements. Our Bayesian method detects two distinct chemodynamic populations with high significance (in logarithmic Bayes factor, $\ln B \sim 33$ ). The metal-rich $\left([\mathrm{Fe} / \mathrm{H}]=-2.05 \pm 0.03\right.$ ) population is kinematically colder (radial velocity dispersion of $\sigma_{v}=$ $\left.4.9_{-1.0}^{+0.8} \mathrm{~km} \mathrm{~s}^{-1}\right)$ and more centrally concentrated than the metal-poor $\left([\mathrm{Fe} / \mathrm{H}]=-2.29_{-0.06}^{+0.05}\right)$ and kinematically hotter population $\left(\sigma_{v}=11.5_{-0.8}^{+0.9} \mathrm{~km} \mathrm{~s}^{-1}\right)$. Furthermore, we apply the same analysis to an independent Multiple Mirror Telescope (MMT)/Hectochelle data set and confirm the existence of two chemodynamic populations in UMi. In both data sets, the metal-rich population is significantly flattened $(\epsilon=0.75 \pm 0.03)$ and the metal-poor population is closer to spherical $\left(\epsilon=0.33_{-0.09}^{+0.12}\right)$. Despite the presence of two populations, we are able to robustly estimate the slope of the dynamical mass profile. We found hints for prolate rotation of order $\sim 2 \mathrm{~km} \mathrm{~s}^{-1}$ in the MMT data set, but further observations are required to verify this. The flattened metal-rich population invalidates assumptions built into simple dynamical mass estimators, so we computed new astrophysical dark matter annihilation $(J)$ and decay profiles based on the rounder, hotter metal-poor population and inferred $\log _{10}\left(J(0.5) / \mathrm{GeV}^{2} \mathrm{~cm}^{-5}\right) \approx$ 19.1 for the Keck data set. Our results paint a more complex picture of the evolution of UMi than previously discussed.
\end{abstract}

Key words: galaxies: evolution-galaxies: kinematics and dynamics-Local Groupcosmology: dark matter-galaxies: individual: Ursa Minor dSph.

\section{INTRODUCTION}

The distribution of dark matter within galaxies is a key test for the $\Lambda \mathrm{CDM}$ (cosmological constant + cold dark matter) cosmological model. Dark matter-only simulations predict that dark matter haloes

^E-mail: apace@ andrew.cmu.edu have cuspy inner density slopes that scale as $\rho_{\mathrm{DM}} \sim r^{-1}$ at small radii (Navarro, Frenk \& White 1996b, 1997). Observations of dwarf, spiral, and low surface brightness galaxies infer shallower profiles (e.g. de Blok \& Bosma 2002; Simon et al. 2005; Kuzio de Naray et al. 2006; de Blok et al. 2008; Kuzio de Naray, McGaugh \& de Blok 2008; Oh et al. 2011, 2015; Adams et al. 2014; Relatores et al. 2019). Solutions to this disagreement generally fall into two categories: the inclusion of baryonic effects (e.g. Navarro, Eke \& 
Frenk 1996a; Governato et al. 2010, 2012; Peñarrubia et al. 2012; Pontzen \& Governato 2012; Brooks et al. 2013; Oñorbe et al. 2015) or a dark matter model differing from the canonical cold and noninteracting model (e.g. recent work by Peter et al. 2013; Rocha et al. 2013; Abazajian 2014; Horiuchi et al. 2014; Kaplinghat et al. 2014; Lovell et al. 2014; Wang et al. 2014; Kaplinghat, Tulin \& Yu 2016; Kamada et al. 2017). There is ongoing debate in the literature over the validity of both solutions. The Milky Way (MW) dwarf spheroidal galaxies (dSphs) have low stellar masses and are highly dark matter-dominated systems (McConnachie 2012; Simon 2019). Accordingly they are excellent laboratories to distinguish between these solutions.

The MW dSphs are close enough for photometric and spectroscopic analysis of individual stars. Analysis of colour-magnitude diagrams (CMDs) has revealed that the brighter 'classical' dSphs $\left(L_{V} \gtrsim 10^{5} \mathrm{M}_{\odot}\right.$ ) have extended star formation histories (Weisz et al. 2014). The spatial distributions of different stellar populations in dSphs may vary as a function of age or metallicity, depending on the dynamical history of the galaxy and the evolution of its gas distribution over time. For example, in similar galaxies red horizontal branch (HB; younger and more metal-rich) stars are generally more centrally concentrated than the older blue HB stars (Harbeck et al. 2001). Large spectroscopic surveys with accurate velocity and metallicity measurements have shown that the stellar kinematics is distinct between the metal-poor and metalrich populations (e.g. Tolstoy et al. 2004; Battaglia et al. 2006, 2008; McConnachie, Peñarrubia \& Navarro 2007; Walker \& Peñarrubia 2011; Hendricks et al. 2014; Kordopatis et al. 2016).

The MW dSphs are dispersion-supported, gas-free systems and among the closest objects for which the motions of individual stars can be utilized for dynamical analysis. Unfortunately, a direct inference of the inner mass slope is hampered by the degeneracy between the mass profile and stellar anisotropy. One promising approach to breaking this degeneracy in the dSphs is to utilize the dynamics of multiple chemodynamic stellar populations (Battaglia et al. 2008; Walker \& Peñarrubia 2011; Read \& Steger 2017).

Thus far, the dynamics of multiple stellar populations has been utilized in three dSphs: Fornax (Walker \& Peñarrubia 2011; Amorisco, Agnello \& Evans 2013), Sculptor (Battaglia et al. 2008; Walker \& Peñarrubia 2011; Agnello \& Evans 2012; Amorisco \& Evans 2012b; Zhu et al. 2016; Strigari, Frenk \& White 2017), and Carina (Hayashi et al. 2018) to infer the mass slope of the dark matter halo. Most results favour a 'cored' halo over 'cuspy' halo, but whether the 'cuspy' solution is excluded (Walker \& Peñarrubia 2011; Agnello \& Evans 2012; Amorisco \& Evans 2012b), just disfavoured (Battaglia et al. 2008; Amorisco et al. 2013) or consistent (Strigari et al. 2017) is under debate. Finding additional dSphs with multiple chemodynamic populations will assist in determining whether the inner dark matter profile in dSphs is a 'cusp' or a 'core'.

In this paper, we present results from a Keck/Deep Imaging Multi-Object Spectrograph (DEIMOS) spectroscopic survey of the Ursa Minor (UMi) dSph that shows, with high significance, two chemodynamic stellar populations. In Section 2, we discuss the observations, data reduction, velocity and metallicity measurements, colour-magnitude selection, and final catalogue selection. In Section 3, we present our statistical methodology, likelihood, and methods to separate foreground MW stars and separate and identify multiple stellar populations. In Section 4, we present our main results on the properties of the chemodynamic populations of $\mathrm{UMi}$, verify our chemodynamic results with an independent Multiple Mirror Telescope (MMT)/Hectochelle data set (Spencer et al. 2018), and search for stellar rotation. In Section 5, we discuss the inner slope of the mass profile. In Section 6, we compare our results in UMi to other dSphs. In Section 7, we compute the astrophysical components for studies of the indirect detection of dark matter. In Section 8 , we conclude and summarize our results.

\section{DATA}

\subsection{Observations and target selection}

Spectroscopic observations were carried out on 2009 February 22-23 (first presented by Kirby et al. 2010), 2010 May 11-12 (first presented by Kirby et al. 2018), and 2012 April 20-22 (not previously published) on the Keck/DEIMOS spectrograph (Faber et al. 2003). All these observations used the $1200 \mathrm{G}$ diffraction grating, which has a groove spacing of $1200 \mathrm{~mm}^{-1}$ and a blaze wavelength of $7760 \AA$. The grating was tilted such that the typical central wavelength of a spectrum was $7800 \AA$, and the typical wavelength range was about $2600 \AA$. In practice, the wavelength range for each spectrum varied by up to $300 \AA$ depending on the location of the slit along the dispersion axis. The grating was used in first order, and higher orders were blocked with the OG550 filter. DEIMOS has a flexure compensation system that keeps the wavelength calibration stable to within $\sim 0.1$ A over a full night. The spectral resolution is $\Delta(\lambda) \sim 1.2 \AA$, which translates to $R \sim 7000$ at the Ca triplet around $8500 \AA$. A summary of our observations is provided in Table 1.

Table 1. Observation log.

\begin{tabular}{lccccc}
\hline Slitmask & No. of targets & Date & Airmass & Seeing $(\operatorname{arcsec})$ & Exposures (s) \\
\hline uss-1 & 68 & 2012 April 19 & 1.58 & 1.1 & $3 \times 1020$ \\
& 68 & 2012 April 23 & 1.60 & 0.8 & $1 \times 1020$ \\
uss-2 & 57 & 2012 April 19 & 1.74 & 1.0 & $2 \times 1020,600$ \\
uss-3 & 74 & 2012 April 21 & 1.55 & 0.5 & $3 \times 960$ \\
uss-4 & 66 & 2012 April 21 & 1.70 & 0.7 & $3 \times 960,480$ \\
uss-5 & 27 & 2012 April 21 & 1.49 & 0.5 & $2 \times 960$ \\
uss-6 & 13 & 2012 April 22 & 1.49 & 0.7 & $2 \times 960,900$ \\
uss-7 & 17 & 2012 April 23 & 1.49 & 0.9 & $2 \times 1020$ \\
uss-8 & 57 & 2012 April 22 & 1.56 & 0.9 & $1 \times 1080,1170$ \\
uss-9 & 24 & 2012 April 23 & 1.55 & 0.7 & $3 \times 1020$ \\
uss-10 & 65 & 2012 April 22 & 1.47 & 0.8 & $3 \times 960$ \\
uss-11 & 56 & 2012 April 21 & 1.48 & 0.5 & $3 \times 1020$ \\
uss-12 & 54 & 2012 April 23 & 1.47 & 0.9 & \\
\hline
\end{tabular}


Spectroscopic targets were selected from various photometric catalogues. Where the slitmask design constraints forced a choice among multiple candidates, we prioritized stars on the red giant branch (RGB). Kirby et al. (2010) described the slitmask design for the 2009 observations. Targets were selected from Bellazzini et al.'s (2002) photometric catalogue in the $V$ and $I$ filters. We used the CMD in conjunction with Yonsei-Yale isochrones (Demarque et al. 2004) to inform the selection. Targets were selected between a blue bound and red bound. The blue bound was 0.1 mag bluer in dereddened $(V-I)_{0}$ than a $2 \mathrm{Gyr}$ isochrone with $[\mathrm{Fe} / \mathrm{H}]=-3.76$ and $[\alpha / \mathrm{Fe}]=0.0$. The red bound was a $14 \mathrm{Gyr}$ isochrone with $[\mathrm{Fe} / \mathrm{H}]=+0.05$ and $[\alpha / \mathrm{Fe}]=+0.3$. HB stars were also selected from a box in the CMD: $20.5>I_{0}>19.0$ and $-0.20<(V-I)_{0}<$ 0.65 . Brighter stars were given higher priority.

Slitmasks from the 2010 observations were designed from Sloan Digital Sky Survey (SDSS) ugriz photometry (Abazajian et al. 2009). Stars were selected to lie within a colour range around a 14.1 Gyr, $[\mathrm{Fe} / \mathrm{H}]=-1.63$, Padova isochrone (Girardi et al. 2004). The allowed range was 0.4 mag bluer and 0.3 mag redder in $(g-r)_{0}$ colour. We also selected HB stars from a box in the CMD: $21>r_{0}>$ 20 and $-0.4<(g-r)_{0}<0.0$. As for the 2009 observations, brighter stars were given higher priorities for spectroscopic selection.

Because there is no published photometric data set for UMi that covers the full extent of the galaxy and is sufficiently deep for our purposes, the spectroscopic target selection for the 2012 observing run relied on a number of different sources.

UMi has been observed by the SDSS, but the SDSS Data Release 7 (DR7; Abazajian et al. 2009) coverage nearly bisects the galaxy along its major axis, with the south-east half of the galaxy included but no data in the north-west half. SDSS Data Release 8 (DR8; Aihara et al. 2011a), in contrast, contains several stripes crossing the galaxy from south-east to north-west, with $\sim 20$ arcmin gaps between each stripe. The difference in coverage between DR7 and DR8 is a result of different data quality criteria in the SDSS processing of those data sets (Padmanabhan, private communication), but there is no evidence for systematic photometric errors in either the DR7 or DR8 imaging in this region. We therefore generated a combined SDSS DR7 + DR8 catalogue for UMi, using DR7 measurements where available and DR8 elsewhere. ${ }^{1}$

In addition to SDSS, we used the wide-field Washington/DDO51 photometry of Palma et al. (2003), the deeper VI imaging of Bellazzini et al. (2002) in the centre of the galaxy, and deep, widefield $g r$ imaging covering $1 \mathrm{deg}^{2}$ with the Canada-France-Hawaii Telescope (CFHT)/Megacam from Muñoz et al. (2018a). These catalogues were merged with the SDSS data taking precedence, followed by stars in the Palma et al., Bellazzini et al., and Muñoz et al. catalogues, in that order. For the latter three data sets, we applied zero-point offsets to the astrometry so that the median position differences with respect to SDSS Data Release 9 (DR9) of all stars in common were zero.

Spectroscopic targeting priorities for stars in the SDSS, Bellazzini et al., and Muñoz et al. data sets were determined using $[\mathrm{Fe} / \mathrm{H}]=$ $-2,10$ Gyr isochrone from Dotter et al. (2008). The RGB selection window was defined so as to include all obvious members of UMi near the centre of the galaxy, with more generous colour limits to the blue side of the RGB to allow for unusually metal-poor stars. We

\footnotetext{
${ }^{1}$ DR8 contained an astrometry error of up to 0.25 arcsec for northern targets (Aihara et al. 2011b; Ahn et al. 2012), so for stars in the DR8 photometric catalogue we used the corrected positions provided in the early release of DR9 (Ahn et al. 2012).
}

constructed a similar selection window for HB stars by generating a large number of synthetic HB stars with the online code provided by Dotter et al. (2007) using the same age and metallicity as for the RGB and then fitting a polynomial to determine the luminosity of the $\mathrm{HB}$ as a function of colour. We assigned RGB candidates higher priorities than $\mathrm{HB}$ candidates, with relative priorities determined by magnitude within each category (where preference is given to brighter stars), and then added priority bonuses for stars already confirmed to be UMi members by Palma et al. (2003) or the 2009/2010 Kirby data sets described above. Stars located within the bounds of either of the two possible substructures in UMi identified by Pace et al. (2014) were given the highest priorities, and then slitmasks were placed to ensure full coverage of both substructures.

\subsection{Reductions and measurements}

We reduced the DEIMOS data using the SPEC2D pipeline developed by the DEEP2 team (Cooper et al. 2012; Newman et al. 2013). The pipeline cuts out the spectrally dispersed image of each slit from the raw data. The image is flat-fielded, and a two-dimensional wavelength solution is calculated from an exposure of $\mathrm{Kr}, \mathrm{Ne}, \mathrm{Ar}$, and Xe arc lamps. The typical root-mean-square difference between the known arc line wavelengths and the calculated wavelength solution is $0.015 \AA\left(0.5 \mathrm{~km} \mathrm{~s}^{-1}\right)$. The stellar spectrum is extracted with 'optimal' extraction (Horne 1986) and made into a skysubtracted, wavelength-calibrated, one-dimensional spectrum. We made some improvements to the pipeline appropriate for our purposes. For example, the procedure for defining the extraction window was optimized for extracting unresolved stars rather than extended galaxies (Simon \& Geha 2007). The one-dimensional wavelength arrays were modified with slight offsets in order to align sky emission lines with their known wavelengths. The wavelength arrays were also modified to remove the effect of differential atmospheric refraction perpendicular to the slit.

We measured radial velocities for each star by comparing the spectra with a set of template spectra measured with DEIMOS (observed by Kirby, Simon \& Cohen 2015). The velocities were calculated from the wavelength shift in log space that minimized $\chi^{2}$ between the target and template spectra. This procedure is similar to a cross-correlation (Tonry \& Davis 1979), but it also takes into account variance in the observed spectrum.

We checked each radial velocity measurement by plotting the template spectrum on top of the target spectrum shifted into the rest frame. In several cases, the velocity measurement clearly failed, and the spectra did not line up. The typical cause was an artefact at the edge of the target spectrum. In these cases, we masked out the offending region of the spectrum and repeated the velocity measurement.

Because DEIMOS is a slit spectrograph, miscentring of stars can cause spurious offsets in the wavelength solution, which translate into offsets in the measured radial velocity. We treated this offset as a shift in the zero-point of the radial velocity. We measured the zeropoint by using the observed wavelengths of telluric absorption from the Earth's atmosphere, which should be at rest in the geocentric frame. This is sometimes known as the 'A-band correction' (Sohn et al. 2007). We cross-correlated each of the observed spectra with the spectrum of a hot star observed with DEIMOS by Kirby et al. (2015). The velocity zero-point was taken to be the velocity shift required to align the template spectrum with the telluric absorption features. This zero-point was added to the radial velocity measured from the stellar absorption lines. We inspected each spectrum to 
Table 2. List of Keck/DEIMOS velocity and metallicity measurements. Column (1): ID; column (2): Gaia DR2 Source ID, stars with -1 are not in the Gaia catalogue; column (3): Megacam ID (Muñoz et al. 2018a), stars with -1 are not in the Megacam catalogue; column (4): RA ( ${ }^{\circ}$ ) (J2000); column (5): Dec. $\left({ }^{\circ}\right.$ ) (J2000); column (6): Modified JD (MJD); column (7): $v_{\text {los }}\left(\mathrm{km} \mathrm{s}^{-1}\right)$; column (8): [Fe/H]; column (9): dSph membership; column (10): metal-rich population membership; column (11): comments. CMD = excluded due to location on colour-magnitude diagram. Gaia NM = non-member due to non-zero parallax and/or large proper motion. RRL = RR Lyrae star in Gaia or PS1 catalogue. NA8190 = MW foreground star due to Na I doublet. This table is available in its entirety in the electronic edition of the journal. A portion is reproduced here to provide guidance on form and content.

\begin{tabular}{lcccccccccc}
\hline ID & Gaia DR2 Source ID & Megacam ID & RA $\left(^{\circ}\right)$ & Dec. $\left(^{\circ}\right)$ & MJD & $v_{\text {los }}\left(\mathrm{km} \mathrm{s}^{-1}\right)$ & {$[\mathrm{Fe} / \mathrm{H}]$} & $p_{\mathrm{dSph}}$ & $p_{\mathrm{MR}}$ & Comments \\
\hline$(1)$ & $(2)$ & $(3)$ & $(4)$ & $(5)$ & $(6)$ & $(7)$ & $(8)$ & $(9)$ & $(10)$ & $(11)$ \\
1 & 1645443305863662592 & 33510 & 227.542184 & 67.177103 & 54884.5 & $-222.6 \pm 2.3$ & $-2.57 \pm 0.11$ & 1.00 & 0.00 & \\
2 & 1645448979516115712 & -1 & 227.494207 & 67.272232 & 54884.5 & $-242.7 \pm 2.2$ & $-1.55 \pm 0.10$ & 1.00 & 0.90 & \\
3 & 1645447811285006464 & -1 & 227.537617 & 67.214524 & 54884.5 & $-233.4 \pm 2.2$ & $-1.74 \pm 0.10$ & 1.00 & 0.44 & \\
4 & 1645485263399853696 & -1 & 227.612946 & 67.410042 & 54884.5 & $-250.1 \pm 2.2$ & $-2.07 \pm 0.10$ & 1.00 & 0.75 & \\
5 & 1645449426192720768 & 32845 & 227.580337 & 67.304530 & 54884.5 & $-7.7 \pm 2.2$ & $-1.88 \pm 0.10$ & - & - & CMD; Gaia NM; NA8190 \\
\hline
\end{tabular}
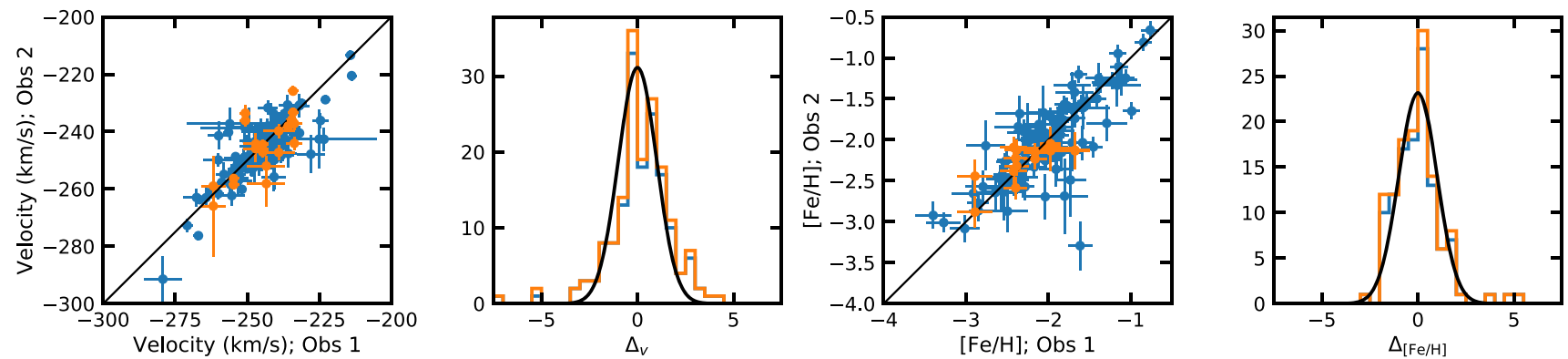

Figure 1. Comparison of velocity (left-hand panels) and $[\mathrm{Fe} / \mathrm{H}]$ (right-hand panels) measurements for stars with multiple measurements. Stars in blue (orange) have two (three) observations. The left- and right-hand velocity panels show the one-to-one comparison and the normalized difference between repeated observations (the left- and right-hand $[\mathrm{Fe} / \mathrm{H}]$ panels are similar). Repeated measurements follow the overlaid normal distribution (mean of zero and variance of one).

ensure that the telluric cross-correlation was valid, just as we did for the stellar absorption. In about a dozen cases where the DEIMOS chip gap fell in the A-band, we had to re-evaluate the telluric crosscorrelation after excluding some pixels around the chip gap.

We calculated velocity errors by resampling the target spectrum 1000 times. In each Monte Carlo trial, we constructed a new spectrum by perturbing the original flux value. The magnitude of the perturbation was sampled from a Gaussian random distribution with a variance equal to the variance estimated for that pixel by SPEC2D. The velocity error was equal to the standard deviation of all of the Monte Carlo trials. Simon \& Geha (2007) found from repeated measurements of the same stars that this statistical error was an incomplete description of the total error. Following their example, we calculated the total error by adding a systematic error of $2.2 \mathrm{~km} \mathrm{~s}^{-1}$ in quadrature with the Monte Carlo statistical error.

We measured metallicities by comparing the continuumnormalized observed spectra to a grid of synthetic spectra. This procedure is identical to that of Kirby, Guhathakurta \& Sneden (2008) and Kirby et al. (2010). We started with a guess at the effective temperature, surface gravity, and metallicity of the star by combining the stars' colours and magnitudes with theoretical isochrones. The temperature and metallicity were allowed to vary to minimize $\chi^{2}$ between the observed spectrum and the synthetic grid. Our measured value of $[\mathrm{Fe} / \mathrm{H}]^{2}$ is the one that minimized $\chi^{2}$.

Errors on $[\mathrm{Fe} / \mathrm{H}]$ were estimated from the diagonal terms of the covariance matrix. This is an incomplete estimate of the error, largely due to covariance with temperature. Kirby et al. (2010)

\footnotetext{
${ }^{2}[\mathrm{Fe} / \mathrm{H}]=\log \frac{n(\mathrm{Fe}) / n(\mathrm{H})}{\mathrm{n}_{\odot}(\mathrm{Fe}) / \mathrm{n}_{\odot}(\mathrm{H})}$, where $n$ is atomic number density.
}

found that adding a systematic error of 0.11 dex in quadrature with the statistical error is an adequate estimate of the error. We adopted the same approach.

Our Keck/DEIMOS measurements are listed in Table 2.

\subsection{Validation}

To verify our measurements we examined stars with repeated measurements (using a cross-match radius of 1 arcsec). We find 155 stars with two spectral measurements and 12 stars with three measurements. In Fig. 1, we compare the repeated line-of-sight velocity and metallicity measurements. We compute the normalized difference, $\Delta$, between measurements. For radial velocity measurements, $\Delta_{v}=\left(v_{1}-v_{2}\right) / \sqrt{\sigma_{\epsilon, v, 1}^{2}+\sigma_{\epsilon, v, 2}^{2}}$, where $v$ and $\sigma_{\epsilon, v}$ correspond to the radial velocity and velocity error, respectively. If the repeated measurements are consistent, the $\Delta$ distribution will follow a Gaussian distribution with a mean of zero and variance of unity. Based on a Shapiro-Wilk test we find that our repeat measurements are consistent with a Gaussian distribution $(p=0.34)$. After removing stars with clear velocity variation, we find $\overline{\Delta_{v}}=0.24$ and $\sigma_{\Delta_{v}}=1.18$ for repeated velocity measurements and $\overline{\Delta_{v}}=-0.15$ and $\sigma_{\Delta_{v}}=1.00$ for repeated metallicity measurements. The second and fourth panels of Fig. 1 show $\Delta$ for the velocity and metallicity measurements, respectively. The tails in the velocity distribution could be due to unresolved binary stars.

To combine velocity measurements we use the weighted mean as the combined radial velocity. For the error we compute the variance of the weighted mean and weighted standard deviation and take the larger of the two for the combined velocity error. 

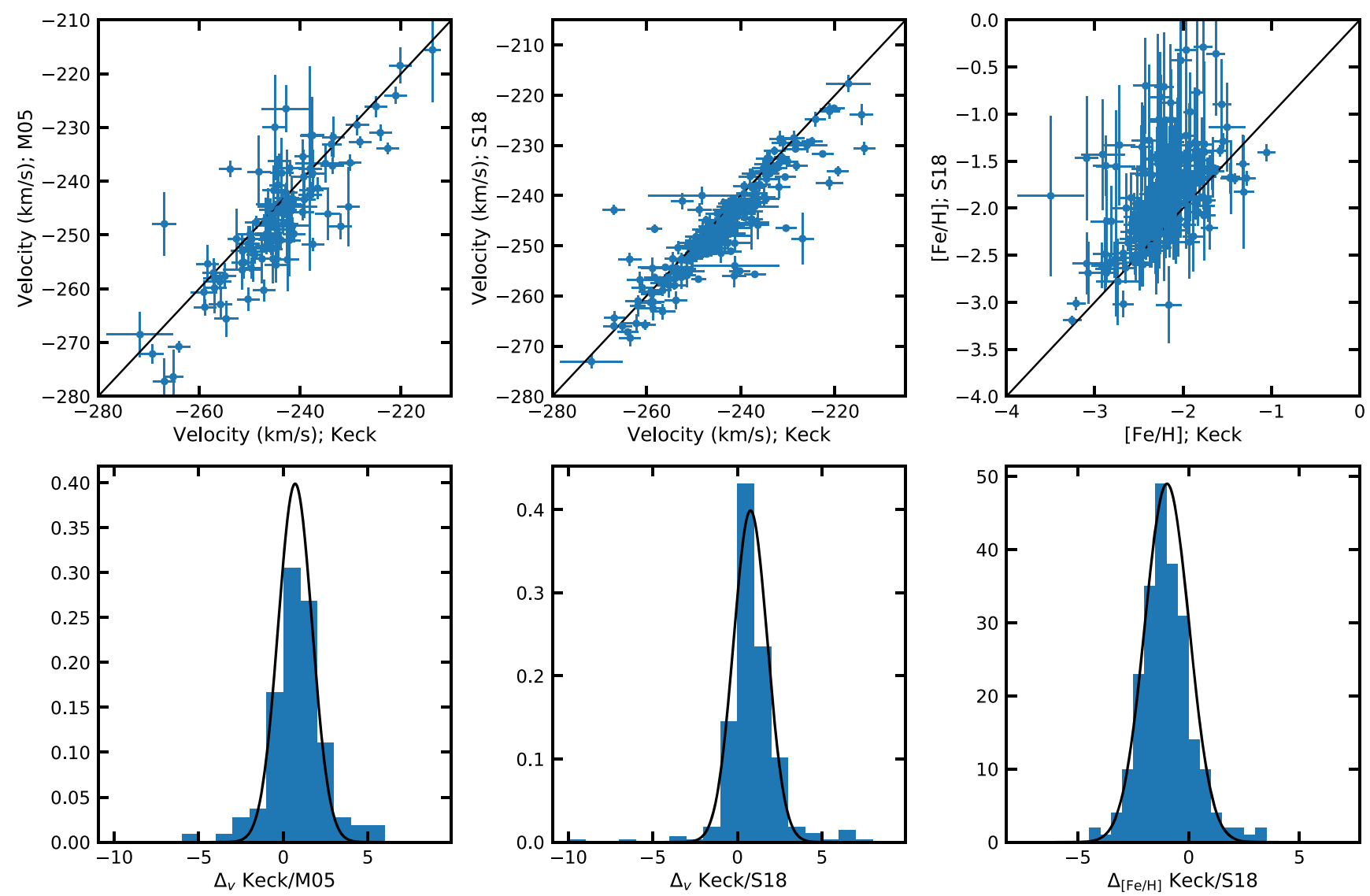

Figure 2. Top: comparison of our velocity and metallicity measurements to previous works: M05 (Muñoz et al. 2005) and S18 (Spencer et al. 2018). Bottom: normalized velocity $\left(\Delta_{v}\right)$ and metallicity $\left(\Delta_{[\mathrm{Fe} / \mathrm{H}]}\right)$ differences between our analysis and previous studies. Overlaid are normal distributions with different means $(\bar{\Delta}=+0.70,+0.77,-0.98)$ but the same dispersion $\left(\sigma_{\Delta}=1\right)$. The velocity and metallicity measurements have zero-point offsets between the different studies. All three distributions are consistent with a spread of one indicating that the relative errors are consistent between studies.

When the weighted standard deviation is larger than the variance of the weighted mean, the star may be variable in velocity and we use the weighted standard deviation to be conservative. For metallicity measurements we use the weighted mean and variance of the weighted mean for all combined measurements.

We compare our velocity and metallicity measurements to two other large UMi spectroscopic samples (Fig. 2). Muñoz et al. (2005) presented velocity measurements from a combination of Keck/High Resolution Echelle Spectrometer (HIRES) and William Herschel Telescope (WHT)/Wide-field Fibre Optic Spectrograph (WYFFOS; the latter were originally presented in Wilkinson et al. 2004). Previous analysis of this data set motivated our target selection (Pace et al. 2014). Spencer et al. (2018) utilized MMT/Hectochelle to measure velocities and metallicities in UMi. There are 108 and 277 stars in common with our data set and Muñoz et al. (2005) and Spencer et al. (2018), respectively. Overall, we find that our velocities are offset from both studies; the average mean normalized offset (after removing outliers) is $\overline{\Delta_{v}}=0.70, \sigma_{\Delta_{v}}=1.17$ and $\overline{\Delta_{v}}=0.77, \sigma_{\Delta_{v}}=0.92$ for Muñoz et al. (2005) and Spencer et al. (2018), respectively. These offsets are likely caused by zero-point offsets in the radial velocity templates assumed between different analyses. We find that there is an offset in the metallicity between the Spencer et al. (2018) MMT/Hectochelle measurements and find $\overline{\Delta_{[\mathrm{Fe} / \mathrm{H}]}}=-0.98$ and $\sigma_{\Delta_{[\mathrm{Fe} / \mathrm{H}]}}=1.03$. This offset may be due to the differences in measurement techniques or due to the different spectral ranges and resolutions. Based on our UMi analysis, we find these normalized offsets translate to offsets of $\Delta \bar{v} \approx 2.4 \mathrm{~km} \mathrm{~s}^{-1}$ and $\Delta \overline{[\mathrm{Fe} / \mathrm{H}]} \approx$ 0.16 . While there are offsets between the different studies, we find that the errors are consistent between the different studies.

\subsection{Final catalogue selection}

As mentioned in Section 2.1, at the time of our target selection and observations there was not a deep and wide-field public photometric catalogue for UMi. There are now several, including the wide-field CFHT/Megacam data (Muñoz et al. 2018a), Panoramic Survey Telescope and Rapid Response System 1 (Pan-STARRS1; Chambers et al. 2016), and Gaia DR2 (Gaia Collaboration 2018a). We use the $g r$ CFHT/Megacam photometry (Muñoz et al. 2018a) for the majority of the sample and Gaia DR2 photometry ( $G$ and $\left.G_{\mathrm{BP}}-G_{\mathrm{RP}}\right)$ for the brightest stars in the sample that are saturated in the Megacam catalogue $(G<18$, roughly $g \sim 18.75)$. We perform a broad isochrone selection to pick stars with colours and magnitudes consistent with UMi. For the Gaia DR2 photometry, we base on cuts on the selection by Gaia Collaboration (2018b). For the Megacam photometry and stars on the RGB, we select stars within a colour window of $0.12 \mathrm{mag}$ from an $[\mathrm{Fe} / \mathrm{H}]=-2,10 \mathrm{Gyr}$ isochrone (Dotter et al. 2008) and exclude stars fainter than $g=22.5$. For 

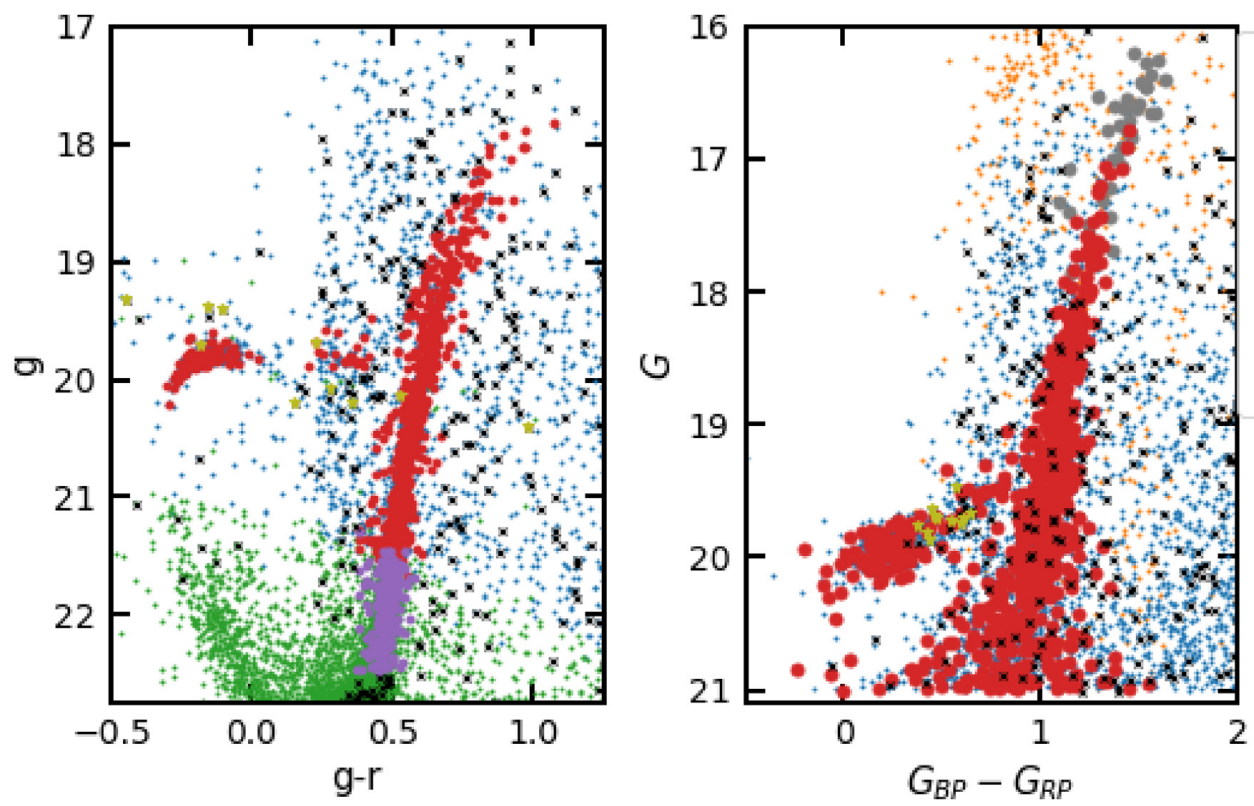

$\begin{array}{ll}\text { - } & \text { Maia only } \\ \text { - Megacam+Gaia } \\ \text { - Gaia candidate } \\ \text { - Both candidate } \\ \text { - Megacam candidate } \\ \text { CMD non-member } \\ \text { * RRL }\end{array}$

Figure 3. Colour-magnitude diagrams (CMDs) in $g-r$ versus $g$ (Megacam; left) and $G_{\mathrm{BP}}-G_{\mathrm{RP}}$ versus $G$ (Gaia; right) of UMi. Blue, orange, and green points show stars that are contained in both catalogues, exclusive to the Gaia catalogue, or exclusive to the Megacam catalogue. Grey, red, and purple stars pass our colour-magnitude selection and are candidate UMi members; grey points utilize Gaia bands, while the reminder use Megacam photometry. Olive stars are RR Lyrae stars identified in PS1 or Gaia catalogues. Stars excluded due to their location on the CMD are shown in black.

the HB selection, we select stars within magnitude windows of 0.3 and 0.2 mag for stars with $g-r<0$ and $0<g-r<0.55$, respectively. The window in the red $\mathrm{HB}$ region is narrower due to the increase in MW interlopers. This selection is similar to the target selection on the preliminary Megacam photometry. Figure 3 shows our isochrone selection for both the Gaia and Megacam photometry.

We use Gaia DR2 proper motions to improve MW foreground identification and determine the proper motion of UMi. We crossmatch our spectroscopic sample with the Gaia DR2 catalogue with a cross-match radius of 1 arcsec (for most stars the cross-match radius is less than 0.5 arcsec) and find 1060 matches out of 1532 stars with an astrometric solution (astrometric_params_solved $=31$ ). We utilize the stellar parallax to identify nearby disc stars. We consider all stars with a non-zero parallax as members of the MW disc $\left(\varpi-3 \sigma_{\varpi}>0\right)$. We calculate the tangential velocity for each star assuming it is at the distance to UMi (Table 3). Any star that would be unbound at UMi's distance $\left(v_{\tan }-3 \sigma_{v_{\text {tan }}}>v_{\text {escape }}\right)$ is considered a nearby MW foreground star (Pace \& Li 2019).

RR Lyrae stars are variable in velocity and not suited for kinematic analysis. We cross-match our sample to the Pan-STARRS RR Lyrae (Sesar et al. 2017) and Gaia DR2 RR Lyrae catalogues (Clementini et al. 2019) and find 17 and 15 matches, respectively (with a 0.5 arcsec cross-match radius). We find a total of 18 RR Lyrae in our spectroscopic sample as 12 stars overlap between the RR Lyrae catalogues. The velocities and locations on a CMD of all 18 stars are consistent with membership in UMi. Two RR Lyrae each has two velocity measurements and exhibit clear velocity variation (Gaia Source ID 1645449593695899264: $-298.3 \pm 2.5$ and $-279.3 \pm 2.4$ and Gaia Source ID 1645468079235094784: $-229.6 \pm 2.6$ and $-235.7 \pm 2.5)$. We exclude all known RR Lyrae from our analysis.

We use the surface gravity sensitive spectral feature at $\mathrm{NaI}$ at $8190 \AA$ to identify additional MW foreground stars (Spinrad \& Taylor 1971; Cohen 1978). Stars with $\sigma_{\epsilon, v}>20 \mathrm{~km} \mathrm{~s}^{-1}$ are excluded
Table 3. Properties of Ursa Minor (UMi) from the literature and selected posterior values from our $\mathrm{dSph}$ and MW mixture model. $r_{\mathrm{p}}, \epsilon, \theta$ assume a Plummer profile, $R_{\mathrm{h}}=r_{\mathrm{p}} \sqrt{1-\epsilon}$.

\begin{tabular}{lll}
\hline Property & \multicolumn{1}{c}{ Value } & \multicolumn{1}{c}{ Reference } \\
\hline$\alpha_{\mathrm{o}}\left({ }^{\circ}\right)$ & 227.2420 & Muñoz et al. (2018b) \\
$\delta_{\mathrm{o}}\left(^{\circ}\right)$ & 67.2221 & Muñoz et al. (2018b) \\
$\theta\left(^{\circ}\right)$ & $50 \pm 1$ & Muñoz et al. (2018b) \\
$\epsilon$ & $0.55 \pm 0.01$ & Muñoz et al. (2018b) \\
$r_{\mathrm{p}}(\operatorname{arcmin})$ & $18.3 \pm 0.11$ & Muñoz et al. (2018b) \\
$R_{\mathrm{h}}(\mathrm{pc})$ & $271 \pm 3$ & Muñoz et al. $(2018 \mathrm{~b})$ \\
$M_{V}$ & $-9.03 \pm 0.05$ & Muñoz et al. $(2018 \mathrm{~b})$ \\
$d(\mathrm{kpc})$ & $76 \pm 5$ & Bellazzini et al. $(2002)$ \\
$\bar{v}\left(\mathrm{~km} \mathrm{~s}^{-1}\right)$ & $-244.7_{-0.3}^{+0.3}$ & This work \\
$\sigma_{v}\left(\mathrm{~km} \mathrm{~s}^{-1}\right)$ & $8.7_{-0.3}^{+0.3}$ & This work \\
$\mu_{\alpha *}\left(\mathrm{mas} \mathrm{yr}^{-1}\right)$ & $-0.151_{-0.014}^{+0.014}$ & This work \\
$\mu_{\delta}\left(\mathrm{mas} \mathrm{yr}^{-1}\right)$ & $0.065_{-0.013}^{+0.013}$ & This work \\
\hline$[\mathrm{Fe} / \mathrm{H}]$ & $-2.13_{-0.02}^{+0.02}$ & This work \\
$\sigma_{[\mathrm{Fe} / \mathrm{H}]}$ & $0.35_{-0.01}^{+0.01}$ & This work \\
$\epsilon$ & $0.59_{-0.02}^{+0.02}$ & This work \\
$r_{\mathrm{p}}(\mathrm{pc})$ & $447_{-20}^{+23}$ & This work \\
$R_{\mathrm{h}}(\mathrm{pc})$ & $287_{-10}^{+11}$ & This work \\
\hline
\end{tabular}

and we do not use the metallicity measurements for stars with $\sigma_{\epsilon,[\mathrm{Fe} / \mathrm{H}]}>0.5$ dex. We compute the $\chi^{2}$ of a non-variable velocity for each star (i.e. any variation is due just to measurement errors) and the corresponding $p$-value. We exclude seven stars with clear indications of velocity variability from our analysis $\left(\Delta_{v}>3\right.$ and/or $p<0.01)$. After our colour-magnitude selection and removing stars with indications of variability, we have 1009 candidate UMi stars. Based on the parallax, large proper motion, or Na I doublet, 64 of these stars are immediately identified as MW foreground stars. 


\section{METHODOLOGY}

To identify dSph members and disentangle chemodynamical populations, we construct mixture models and assess statistical significance with model selection tests. This analysis builds upon the statistical framework of Walker \& Peñarrubia (2011) by extending both the stellar distribution and selection function to axisymmetric systems, by including a MW model, and by including proper motion to significantly improve MW foreground selection. To address the significance of additional populations we compute the Bayes factor between single and multipopulation models.

We work in a Bayesian framework for disentangling different components. The probability of observing a data set, $\boldsymbol{x}=\left\{\boldsymbol{x}_{i}\right\}$, assuming a particular hypothesis or model, $H$, characterized by parameters, $\mathscr{M}$, is given by the likelihood $\mathcal{L}(\boldsymbol{x} \mid \mathscr{M})=\mathcal{P}(\boldsymbol{x} \mid \mathscr{M}, H)$. We are interested in solving for the model parameters, found by determining the posterior distribution, $\mathcal{P}(\mathscr{M} \boldsymbol{x}, H)$. The posterior and likelihood are related via Bayes theorem:

$\mathcal{P}(\mathscr{M} \mid \boldsymbol{x}, H)=\frac{\mathcal{L}(\boldsymbol{x} \mid \mathscr{M}) \operatorname{Pr}(\mathscr{M}, H)}{\mathcal{P}(\boldsymbol{x}, H)}$,

where $\operatorname{Pr}(\mathscr{M}, H)$ is the prior distribution representing any previously known information about the model under consideration, and $\mathcal{P}(\boldsymbol{x}, H)$ is the marginal likelihood, a normalizing factor. The marginal likelihood is commonly referred to as the Bayesian evidence in astrophysics. It is given by

$Z=\mathcal{P}(\boldsymbol{x}, H)=\int_{\mathscr{M}} \mathcal{P}(\boldsymbol{x} \mid \mathscr{M}, H) \operatorname{Pr}(\mathscr{M}) \mathrm{d} \mathscr{M}$.

For general parameter estimation, computing the normalization is unnecessary. However, it is useful for model selection purposes. To evaluate the posterior distribution and evidence we utilize multimodal nested sampling (Skilling 2004; Feroz \& Hobson 2008; Feroz, Hobson \& Bridges 2009). The nested sampling algorithm transforms the multidimensional evidence integral (equation 2) into a one-dimensional integral over the 'prior volume'. The integral is evaluated by sampling the likelihood in a decreasing sequence of prior volumes, assuming that the inverse of the prior volume exists and is a monotonically decreasing function. As a by-product of sampling the likelihood, the posterior is also computed (for a more detailed description, see Feroz \& Hobson 2008; Feroz et al. 2009).

For our analysis, the likelihood at each data point is independent and, therefore, the total likelihood is the product of the likelihood at each data point,

$\mathcal{L}(\boldsymbol{x} \mid \mathscr{M})=\prod_{x=i}^{N} \mathcal{L}\left(\boldsymbol{x}_{i} \mid \mathscr{M}\right)$.

The likelihood at each data point is a mixture of a MW foreground and a dSph population (e.g. Koposov et al. 2011; Martinez et al. 2011; Walker \& Peñarrubia 2011):

$\mathcal{L}\left(\boldsymbol{x}_{i} \mid \mathscr{M}\right)=f_{\mathrm{MW}} \mathcal{L}_{\mathrm{MW}}\left(\boldsymbol{x}_{i} \mid \mathscr{M}_{\mathrm{MW}}\right)+f_{\mathrm{dSph}} \mathcal{L}_{\mathrm{dSph}}\left(\boldsymbol{x}_{i} \mid \mathscr{M}_{\mathrm{dSph}}\right)$,

where $f_{\mathrm{MW} / \mathrm{dSph}}$ denotes the observed fraction of stars within that component and $f_{\mathrm{MW}}+f_{\mathrm{dSph}}=1$. In general, additional components can be added with the constraint: $\sum_{c} f_{c}=1$. This may include additional dSph components (e.g. Amorisco \& Evans 2012b; Kordopatis et al. 2016) or additional foreground components (e.g. the background model for M31 satellites is composed of stars from both the MW and M31 halo; Tollerud et al. 2012; Collins et al. 2013; Gilbert et al. 2018). For brevity, we will drop the parameter denotation $(\mid \mathscr{M})$ from the likelihood arguments. In some of the later analysis, to identify chemodynamic components, we decompose the
dSph likelihood into multiple components,

$\mathcal{L}_{\mathrm{dSph}}\left(\boldsymbol{x}_{i}\right)=f_{1} \mathcal{L}_{1}\left(\boldsymbol{x}_{i}\right)+f_{2} \mathcal{L}_{2}\left(\boldsymbol{x}_{i}\right)$.

To determine a star's membership in a component we compute the ratio of the component likelihood to the total likelihood for that star (e.g. Martinez et al. 2011; Pace et al. 2014). In more concrete terms, the membership probability for the $i$ th star to be in component $c$ is

$p_{c, i}=\frac{f_{c} \mathcal{L}_{c}\left(\boldsymbol{x}_{i}\right)}{\sum_{k} f_{k} \mathcal{L}_{k}\left(\boldsymbol{x}_{i}\right)}$.

We compute the membership from the posterior distribution. Each star will have a probability distribution of membership for each component, for practicality, we use the median membership for derived quantities.

Model selection. To determine whether multiple components are significant we compute the logarithmic Bayes factor, $\ln B$. The Bayes factor compares the relative odds in favouring model A over model $\mathrm{B}$ after examining the data. It is the ratio of the evidences (equation 2) computed for each model (with the assumption that a priori both models are equally favoured),

$B_{\mathrm{AB}}=\frac{\mathcal{P}\left(\boldsymbol{x}, H_{\mathrm{A}}\right)}{\mathcal{P}\left(\boldsymbol{x}, H_{\mathrm{B}}\right)}=\frac{Z_{\mathrm{A}}}{Z_{\mathrm{B}}}$.

It naturally incorporates Occam's razor as larger or more complicated model spaces are penalized. For models $\mathrm{A}$ and $\mathrm{B}, \ln B_{\mathrm{AB}}=\ln B$ $>0$ favours model $\mathrm{A}$ and $\ln B<0$ favours model $\mathrm{B}$. To interpret the significance of the Bayes factor, we follow the empirical Jeffreys' scale. The ranges of $<1,1-2.5,2.5-5$, and $>5$ correspond to regions of inconclusive, weak, moderate, and strong evidence, respectively (see Trotta 2008).

\subsection{Selection function}

Because of limited telescope time, not all spectroscopic candidates can be observed. The spatial distribution of stars with spectroscopic measurements generally does not follow the intrinsic spatial distribution of stars (due to telescope field of view, mask size, etc.). To ensure that spatial parameters of the $\mathrm{dSph}$ can be recovered from the spectroscopic distribution, we compute the selection function, $S(x$, $y$ ). The selection function acts as a mapping between the observed and intrinsic spatial distribution of stars (Wang et al. 2005; Walker \& Peñarrubia 2011).

To construct $S(x, y)$, we smooth the ratio of observed to candidate stars within the UMi region (Walker \& Peñarrubia 2011):

$$
S(x, y)=\frac{\mathrm{d} N_{\mathrm{obs}}(x, y)}{\mathrm{d} N_{\text {cand }}(x, y)} \approx \frac{\sum_{i=1}^{N_{\text {obs }}} \exp \left[-\frac{1}{2} \frac{\left(x_{i}-x\right)^{2}+\left(y_{i}-y\right)^{2}}{k^{2}}\right]}{\sum_{i=1}^{N_{\text {cand }}} \exp \left[-\frac{1}{2} \frac{\left(x_{i}-x\right)^{2}+\left(y_{i}-y\right)^{2}}{k^{2}}\right]},
$$

where $k$ is the smoothing scale (Walker $\&$ Peñarrubia 2011). $N_{\text {cand }}$ are all stars in the UMi region that fall within our photometric selection in Section 2.4, while $N_{\text {obs }}$ are all spectroscopically observed stars within the same photometric selection. We use the projected spatial positions $(x, y)$ instead of the projected radial positions $\left(R=\sqrt{x^{2}+y^{2}}\right)$ because UMi is more aspherical than Fornax and Sculptor $(q \approx 0.45$ compared to $q \approx 0.71$ and 0.67 ; Muñoz et al. 2018b). In addition, the stellar populations may not necessarily have the same ellipticity.

To set $k$, we construct and observe mock data sets with different values of $k$ ranging from 25 to $400 \mathrm{pc}(1-18 \operatorname{arcmin}$ at $d=$ $76 \mathrm{kpc})$. We find that for spherically symmetric systems, the 


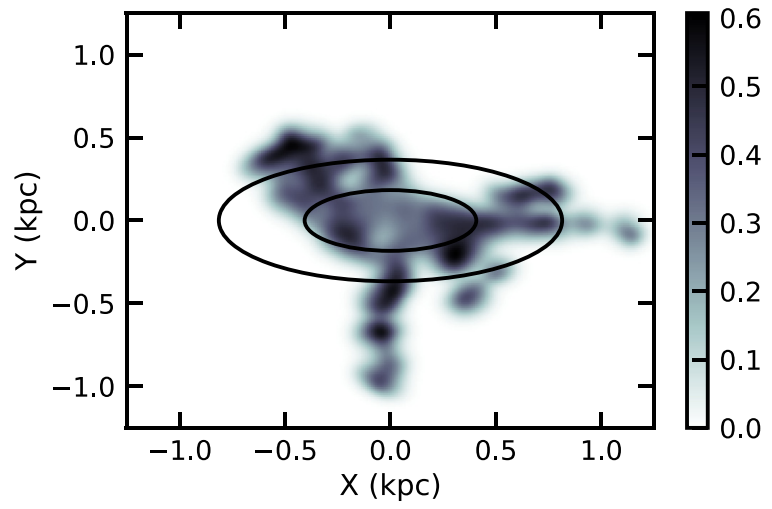

Figure 4. Selection function of Keck/DEIMOS sample (smoothing scale of $k=50 \mathrm{pc}$ ). For reference, we have overlaid ellipses representing the one and two times the half-light radius. The $x$ and $y$ axes are aligned with the major and minor axes of UMi, respectively (position angle of $50^{\circ}$ ).

choice of $k$ does not make an appreciable difference. ${ }^{3}$ Whereas for axisymmetric systems, an incorrect choice in $k$ will strongly bias the recovered structural parameters. Our tests, with a layout approximating the locations and sizes of Keck/DEIMOS masks, show that $50 \leq k \leq 75 \mathrm{pc}(2 \leq k \leq 3 \mathrm{arcmin})$ correctly recovers the input structural parameters. A larger choice of $k$ will bias the structural posteriors. The spatial scale will be underestimated and axial ratio overestimated. The spatial bias increases as $k$ increases.

In Fig. 4, we plot the selection function we use for the UMi analysis with $k=50 \mathrm{pc}$. We align the $x$-axis and the major axis (using a position angle of $\theta=50^{\circ}$; Muñoz et al. 2018b). We fix $k=$ $50 \mathrm{pc}$ for the main analysis.

\subsection{Likelihoods}

This analysis uses line-of-sight velocity $\left(v, \sigma_{\epsilon, v}\right)$, metallicity $\left([\mathrm{Fe} / \mathrm{H}], \sigma_{\epsilon,[\mathrm{Fe} / \mathrm{H}]}\right)$, spatial position $(x, y)$, and proper motion ${ }^{4}\left(\mu_{\alpha *}\right.$, $\left.\sigma_{\epsilon, \mu_{\alpha} *}, \mu_{\delta}, \sigma_{\epsilon, \mu_{\delta}}, C_{\mu_{\alpha *} \mu_{\delta}}\right)$ for each data point..$^{5}$ Our analysis utilizes synthetic measurements of the iron abundance, $[\mathrm{Fe} / \mathrm{H}]$. Other works have utilized different metallicity tracers, for example the Ca II triplet (Battaglia et al. 2008) or the $\Sigma \mathrm{Mg}$ index (Walker \& Peñarrubia 2011).

We assume that probability distributions of the velocity, position, metallicity, and proper motions are independent of one another, therefore, the likelihood of the dSph or MW is

$\mathcal{L}_{\mathrm{dSph} / \mathrm{MW}}=\mathcal{P}^{\mathrm{vel}} \mathcal{P}^{\text {spatial }} \mathcal{P}^{[\mathrm{Fe} / \mathrm{H}]} \mathcal{P}^{\mathrm{PM}}$.

The majority of the probability distributions are assumed to be Gaussian or multivariate Gaussian distributions. For example, the probability distribution of the velocity term is $\mathcal{P}^{\mathrm{vel}}\left(v_{i}, \sigma_{\epsilon, v_{i}} \mid \bar{v}, \sigma_{v}\right)=$ $\mathcal{N}\left(v_{i}-\bar{v}, \sqrt{\sigma_{\epsilon, v, i}^{2}+\sigma_{v}^{2}}\right)$, where $\mathcal{N}(a, b)$ is a normal distribution

\footnotetext{
${ }^{3}$ Walker \& Peñarrubia (2011) reach the same conclusion.

${ }^{4} \mu_{\alpha *}=\mu_{\alpha} \cos \delta=-\mu_{\mathrm{W}}$.

${ }^{5}$ We note that not all stars have metallicity or proper motion measurements. For each data point without a particular measurement that likelihood term must be integrated out (i.e. all possible metallicity or proper motion values are considered). As the individual likelihood terms are normalized to unity, integrating over all possible values drops the term from the likelihood and the membership probability for that star will only consider spatial and velocity information.
}

with the mean $a$ and dispersion $b . \bar{v}$ and $\sigma_{v}$ are the average velocity and velocity dispersion, respectively.

For an extended object, like a dSph, the average velocity is a function of spatial position due to projection effects, sometimes referred to as the perspective motion, and we replace $\bar{v}$ with $v_{\text {rel }}(\alpha$, $\delta$ ) (Feast, Thackeray \& Wesselink 1961; van der Marel et al. 2002; Kaplinghat \& Strigari 2008; Walker, Mateo \& Olszewski 2008). The effect can be understood as the difference between the $z$ coordinate and line-of-sight direction, for example, $v_{\text {rel }}(x, y) \approx-v_{z}+v_{x} x / d$ $+v_{y} y / d$ (Kaplinghat \& Strigari 2008). Our implementation of the perspective motion follows the appendix of Walker et al. (2008). For UMi, the effect is $\Delta|v| \sim 0.1-0.2 \mathrm{~km} \mathrm{~s}^{-1}$.

The bright dSphs have flat isothermal line-of-sight velocity dispersions (Muñoz et al. 2005, 2006; Walker et al. 2007), therefore, we initially assume $\sigma_{v}$ is constant with radius. However, this assumption may not hold for individual chemodynamic components (Battaglia et al. 2008; Strigari et al. 2017); we address this in Section 4. We assume the metallicity likelihood is a Gaussian distribution with a free mean, $\overline{[\mathrm{Fe} / \mathrm{H}]}$, and dispersion, $\sigma_{[\mathrm{Fe} / \mathrm{H}]}$.

The likelihood for the spatial distribution is (Wang et al. 2005; Walker \& Peñarrubia 2011)

$\mathcal{P}^{\text {spatial }}\left(x_{i}, y_{i}\right)=\frac{S\left(x_{i}, y_{i}\right) \Sigma\left(x_{i}, y_{i}\right)}{\int S(x, y) \Sigma(x, y) \mathrm{d} A}$,

where $\Sigma(x, y)$ is the projected stellar distribution and $S(x, y)$ is the selection function (Section 3.1). The denominator ensures that the positional likelihood is normalized and acts as a weight for spatial profile reconstruction. We model the projected stellar distribution with an elliptical Plummer profile (Plummer 1911),

$\Sigma(x, y)=\frac{1}{(1-\epsilon) \pi r_{\mathrm{p}}^{2}} \frac{1}{\left(1+R_{\mathrm{e}}^{2} / r_{\mathrm{p}}^{2}\right)^{2}}$,

where $R_{\mathrm{e}}=\left(x^{2}+y^{2} /(1-\epsilon)^{2}\right)$ is the elliptical radius, $r_{\mathrm{p}}$ is the stellar scale radius, $q$ is the axial ratio, and $\epsilon$ is the ellipticity. For this analysis we will approximate the spherically averaged half-light radius as $R_{\mathrm{h}}=r_{\mathrm{p}} \sqrt{1-\epsilon}$. We use a Plummer profile for simplicity but note that stellar distribution profiles with additional parameters may provide better fits (e.g. Sérsic; Muñoz et al. 2018b).

We model the proper motion likelihood as a multivariate Gaussian due to the correlated error between the $\mu_{\alpha *}$ and $\mu_{\delta}$ components (Pace \& Li 2019). The proper motion likelihoods have free means, $\overline{\mu_{\alpha *}}$ and $\overline{\mu_{\delta}}$, and dispersions, $\sigma_{\mu_{\alpha *}}$ and $\sigma_{\mu_{\delta}}$. For the dSph component we fix $\sigma_{\mu_{\alpha *}}=\sigma_{\mu_{\delta}}=10 \mathrm{~km} \mathrm{~s}^{-1}=0.03 \mathrm{mas} \mathrm{yr}^{-1}$ and leave the MW dispersions as free parameters. The proper motion errors for the brightest UMi stars are $\approx 40 \mathrm{~km} \mathrm{~s}^{-1}$ and rapidly increase for fainter stars. Because of the large errors it is not possible to infer the intrinsic dSph proper motion dispersions but this may be possible in future Gaia data releases. The expected MW halo dispersion will be $\approx 100-200 \mathrm{~km} \mathrm{~s}^{-1}$ depending on the component (e.g. disc, halo).

The individual MW probability distributions are similar to the $\mathrm{dSph}$ components. We assume Gaussian or multivariate Gaussians for the velocity, metallicity, and proper motion components with free means $\left(\bar{v}_{\mathrm{MW}}, \overline{[\mathrm{Fe} / \mathrm{H}}_{\mathrm{MW}}, \overline{\mu_{\alpha *, \mathrm{MW}}}, \overline{\mu_{\delta, \mathrm{MW}}}\right)$ and variances $\left(\sigma_{v, \mathrm{MW}}\right.$, $\left.\sigma_{[\mathrm{Fe} / \mathrm{H}], \mathrm{MW}}, \sigma_{\mu_{\alpha *}, \mathrm{MW}}, \sigma_{\mu_{\delta}, \mathrm{MW}}\right)$. For the spatial distribution we assume that it is uniform within the UMi region. After accounting for the selection function the spatial probability distribution is $\mathcal{P}_{\mathrm{MW}}^{\text {spatial }}\left(x_{i}, y_{i}\right)=S\left(x_{i}, y_{i}\right) / \int S(x, y) \mathrm{d} A$.

There are a number of stars that can be immediately identified as foreground stars due to the surface gravity sensitive spectral feature at Na I at $8190 \AA$, a non-zero parallax, and/or an extremely large tangential velocity (see Section 2.4). We do not want to immediately remove these stars from the sample as their $v$ and $[\mathrm{Fe} / \mathrm{H}]$ will help 
Table 4. Posterior distributions for one and two population models for the RGB sample. The 1 and 2 labels refer to the metal-rich and metal-poor populations, respectively. The second and third column are results with the Keck/DEIMOS data set and the last two columns are results with the MMT/Hechocelle data set (Spencer et al. 2018). Note that $R_{\mathrm{h}}=r_{\mathrm{p}} \sqrt{1-\epsilon}$.

\begin{tabular}{lcccc}
\hline Parameter & One (Keck) & Two (Keck) & One (MMT) & Two (MMT) \\
\hline $\bar{v}\left(\mathrm{~km} \mathrm{~s}^{1}\right)$ & $-244.7_{-0.4}^{+0.4}$ & $-244.7_{-0.3}^{+0.3}$ & $-247.0_{-0.4}^{+0.4}$ & $-246.9_{-0.4}^{+0.4}$ \\
$\mu_{\alpha *}$ & $-0.149_{-0.014}^{+0.014}$ & $-0.149_{-0.014}^{+0.014}$ & $-0.150_{-0.012}^{+0.012}$ & $-0.150_{-0.012}^{+0.012}$ \\
$\mu_{\delta}$ & $0.064_{-0.013}^{+0.013}$ & $0.064_{-0.013}^{+0.013}$ & $0.053_{-0.011}^{+0.011}$ & $0.053_{-0.011}^{+0.011}$ \\
$\sigma_{v, 1}\left(\mathrm{~km} \mathrm{~s}^{-1}\right)$ & $8.6_{-0.3}^{+0.3}$ & $4.9_{-1.0}^{+0.8}$ & $8.6_{-0.3}^{+0.3}$ & $7.3_{-0.6}^{+0.5}$ \\
$\sigma_{v, 2}\left(\mathrm{~km} \mathrm{~s}^{-1}\right)$ & - & $11.5_{-0.8}^{+0.9}$ & - & $11.7_{-1.0}^{+1.2}$ \\
$\overline{[\mathrm{Fe} / \mathrm{H}]_{1}}$ & $-2.15_{-0.02}^{+0.01}$ & $-2.05_{-0.03}^{+0.03}$ & $-2.02_{-0.02}^{+0.02}$ & $-1.90_{-0.03}^{+0.03}$ \\
$\overline{[\mathrm{Fe} / \mathrm{H}]_{2}}$ & - & $-2.29_{-0.06}^{+0.05}$ & - & $-2.41_{-0.11}^{+0.10}$ \\
$\sigma_{[\mathrm{Fe} / \mathrm{H}], 1}$ & $0.34_{-0.01}^{+0.01}$ & $0.26_{-0.03}^{+0.02}$ & $0.37_{-0.02}^{+0.02}$ & $0.26_{-0.02}^{+0.02}$ \\
$\sigma_{[\mathrm{Fe} / \mathrm{H}], 2}$ & - & $0.36_{-0.03}^{+0.03}$ & - & $0.35_{-0.04}^{+0.05}$ \\
$r_{\mathrm{p}, 1}(\mathrm{pc})$ & $449_{-24}^{+27}$ & $444_{-37}^{+42}$ & $450_{-30}^{+33}$ & $425_{-38}^{+45}$ \\
$r_{\mathrm{p}, 2}(\mathrm{pc})$ & - & $457_{-46}^{+58}$ & - & $582_{-121}^{+185}$ \\
$\epsilon_{1}$ & $0.60_{-0.03}^{+0.02}$ & $0.75_{-0.03}^{+0.03}$ & $0.56_{-0.03}^{+0.03}$ & $0.64_{-0.04}^{+0.04}$ \\
$\epsilon_{2}$ & - & $0.33_{-0.12}^{+0.09}$ & - & $0.21_{-0.14}^{+0.16}$ \\
$R_{\mathrm{h}, 1}(\mathrm{pc})$ & $286_{-12}^{+13}$ & $221_{-17}^{+17}$ & $297_{-15}^{+15}$ & $253_{-17}^{+18}$ \\
$R_{\mathrm{h}, 2}(\mathrm{pc})$ & - & $374_{-37}^{+49}$ & - & $512_{-97}^{+145}$ \\
$f_{1}$ & - & $0.54_{-0.10}^{+0.09}$ & - & $0.76_{-0.09}^{+0.07}$ \\
\hline & & &
\end{tabular}

construct the MW foreground distribution and assist in identifying MW stars without Gaia astrometric measurements. These stars are only used in the MW likelihood and we do not include their proper motion information in the likelihood.

\section{RESULTS}

\subsection{Ursa Minor properties}

We first explore a model with a single dSph component to identify UMi and foreground MW stars. The centre, position angle, and distance are fixed to literature measurements and the adopted values are listed in Table 3. There are 1009 stars that pass our cuts and are used as input in the mixture model. Stars identified as MW stars based on their parallax, Na I doublet, or large proper motions are fixed to the MW component and only their velocity and metallicity information are included in the mixture model.

We find $\bar{v}=-244.7 \pm 0.3 \mathrm{~km} \mathrm{~s}^{-1}$ and $\sigma_{v}=8.7 \pm 0.3 \mathrm{~km} \mathrm{~s}^{-1}$ for UMi. The velocity dispersion is consistent with $\sigma_{v}=9.5 \pm$ $1.2 \mathrm{~km} \mathrm{~s}^{-1}$ (Walker et al. 2009) and in between the measurements of $\sigma_{v}=8.0 \pm 0.3 \mathrm{~km} \mathrm{~s}^{-1}$ (Spencer et al. 2018) ${ }^{6}$ and $\sigma_{v}=11.5 \pm 0.6$ (Muñoz et al. 2005). The Muñoz et al. (2005) data set is more spatially extended and is a combination of WHT/WYFFOS (Wilkinson et al. 2004) and Keck/HIRES observations. The difference in $\sigma_{v}$ could be due to a $\sigma_{v}$ that increases with radius, or the Muñoz et al. (2005) data set could consist of a larger fraction of stars in the kinematically hotter population (see next section). Another possibility

${ }^{6}$ We note that in our analysis of the Spencer et al. (2018) data, we find a larger $\sigma_{v}$ value that is consistent with our Keck/DEIMOS results. For further details see Section 4.3. is a velocity offset between the two instruments that inflates $\sigma_{v}$. The metallicity properties we find are $\overline{[\mathrm{Fe} / \mathrm{H}]}=-2.13 \pm 0.02$ and $\sigma_{[\mathrm{Fe} / \mathrm{H}]}=0.35 \pm 0.01$ that agree with $\overline{[\mathrm{Fe} / \mathrm{H}]}=-2.13 \pm 0.01$ and $\sigma_{[\mathrm{Fe} / \mathrm{H}]}=0.34$ from Kirby et al. (2011). The spatial properties we derive assuming a Plummer distribution are $r_{\mathrm{p}}=447_{-20}^{+23} \mathrm{pc}, \epsilon=$ $0.59 \pm 0.02$, and $R_{\mathrm{h}}=287_{-10}^{+11} \mathrm{pc}\left(R_{\mathrm{h}}=r_{\mathrm{p}} \sqrt{1-\epsilon}\right)$. This agrees with $q=0.45 \pm 0.01$ and $R_{\mathrm{h}}=271 \pm 3$ pc derived from deep, widefield photometric data (Muñoz et al. 2018b). Our proper motion measurement agrees within the errors with previous Gaia DR2 measurements (Fritz et al. 2018; Gaia Collaboration 2018b).

Of the 1009 candidate stars, 892 (898) have membership greater than $>0.95$ ( $>0.90)$. The inclusion of Gaia proper motions results in very few stars with intermediate membership; only 12 stars have membership values in the range $0.05<p_{\mathrm{dSph}}<0.90$ (only one of which has a Gaia DR2 astrometric solution). Overall, we find our metallicity, spatial, and proper motion properties of UMi are consistent with previous work, whereas our velocity dispersion measurement is in moderate tension $(\sim 2 \sigma-3 \sigma)$ with both Muñoz et al. (2005) and Spencer et al. (2018) but lies in between these measurements.

\subsection{Detection of two chemodynamical stellar populations}

In our search for multiple chemodynamic populations, we use RGB UMi members with metallicity measurements $\left(N=679 ; p_{\mathrm{dSph}}>\right.$ 0.95). Metallicity measurements are key for this analysis and HB stars may not have as robust metallicity measurements. To separate the populations, we set a prior between the velocity dispersions $\left(\sigma_{v, 1}<\sigma_{v, 2}\right)$ and do not assume any additional priors between the metallicity and/or spatial parameters. We assume there is no offset in mean line-of-sight velocity or proper motion between the two 


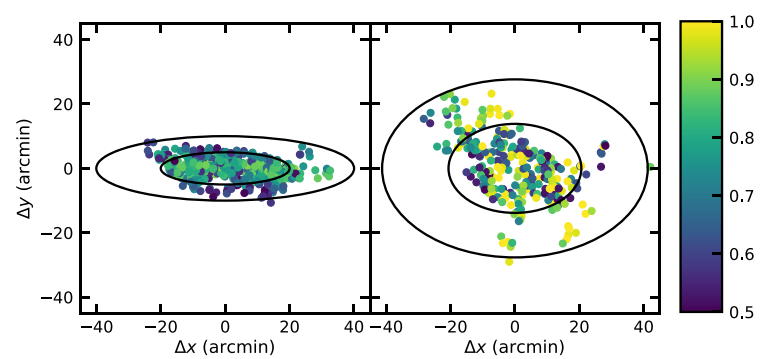

Figure 5. Spatial distribution of stars in the metal-rich (left) and metalpoor (right) chemodynamic populations. Stars are assigned based on the population in which they have larger membership probability. Ellipses with one and two times the half-light radius are shown. The metal-rich population is significantly more elliptical than the metal-poor population.

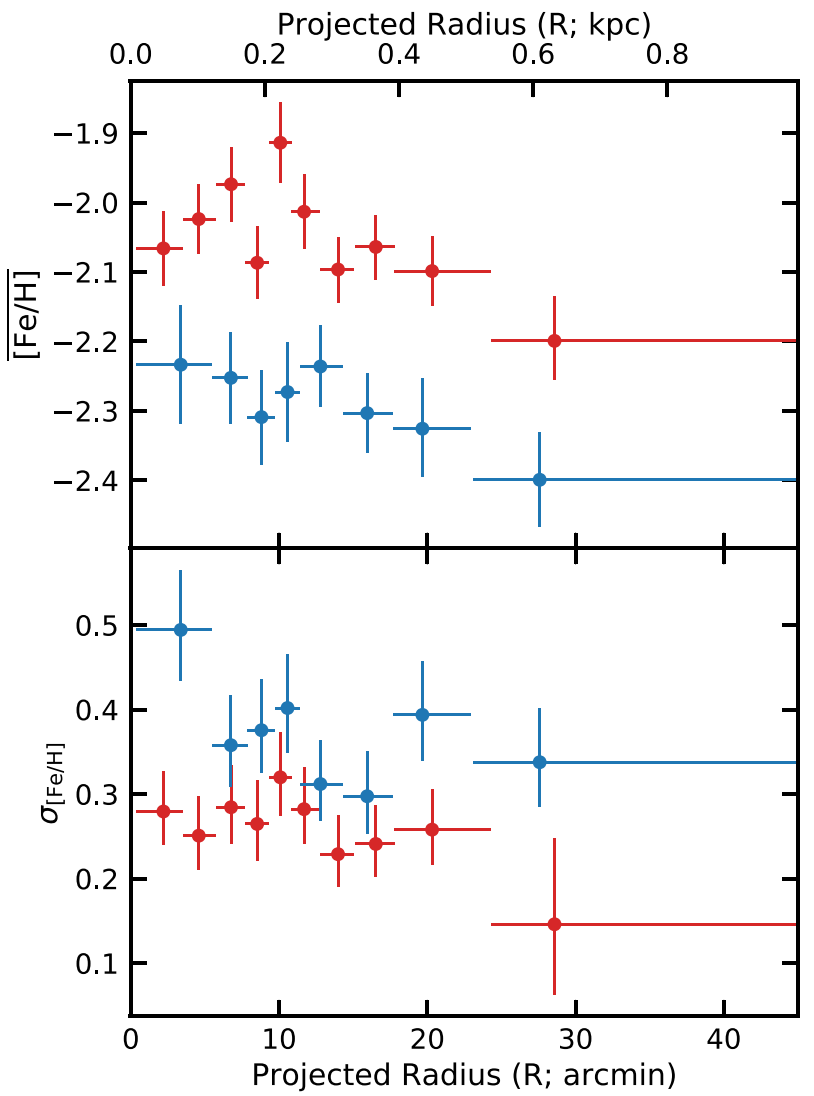

Figure 6. Radial distribution of the mean metallicity (top; $\overline{[\mathrm{Fe} / \mathrm{H}]}$ ) and metallicity dispersion (bottom; $\sigma_{[\mathrm{Fe} / \mathrm{H}]}$ ) for the metal-rich (red) and metalpoor (blue) populations. Each bin contains enough stars such that $\sum p_{i}=$ 50 . The spatial error bars represent the radial extent of stars within each bin, while the velocity dispersion errors are the 68 per cent confidence intervals.

populations. The posteriors of the two population analysis are listed in Table 4 along with results from single component analysis with the same subset of RGB stars.

We find, with high significance $(\ln B=33.47)$, two populations with distinct chemical, kinematic, and spatial distributions. The first population is kinematically cold $\left(\sigma_{v}=4.9_{-1.0}^{+0.8} \mathrm{~km} \mathrm{~s}^{-1}\right)$, more metal-rich $(\overline{[\mathrm{Fe} / \mathrm{H}]}=-2.05 \pm 0.03)$, and centrally concentrated $\left(R_{\mathrm{h}}=221_{-17}^{+17} \mathrm{pc}\right)$. The second population is kinematically hot $\left(\sigma_{v}=11.5_{-0.8}^{+0.9} \mathrm{~km} \mathrm{~s}^{-1}\right)$, more metal-poor $\left(\overline{[\mathrm{Fe} / \mathrm{H}]}=-2.29_{-0.06}^{+0.05}\right)$, and spatially more extended $\left(R_{\mathrm{h}}=374_{-37}^{+49} \mathrm{pc}\right)$. The chemodynami-

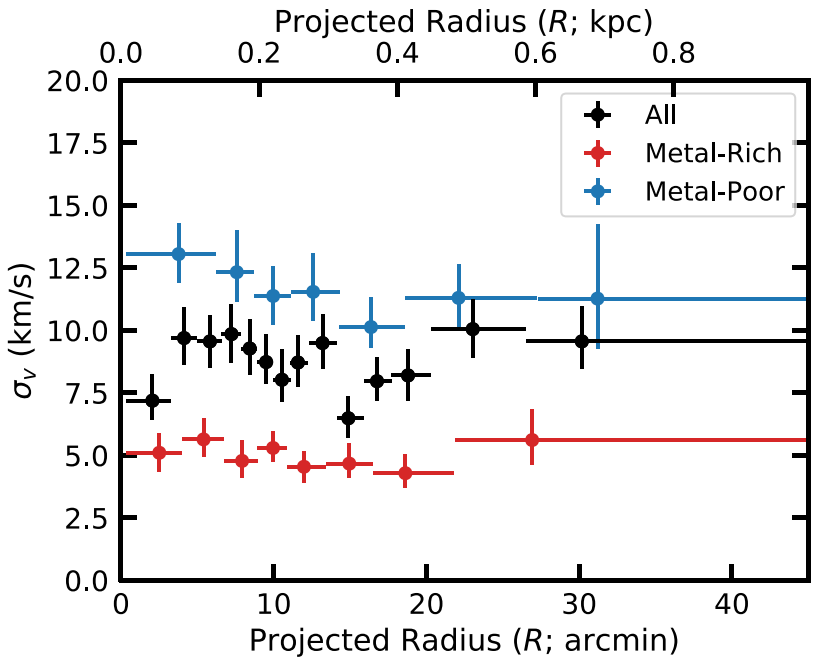

Figure 7. Velocity dispersion $\left(\sigma_{v}\right)$ in radial bins. The average velocity is fixed to the best-fitting UMi value. The results are shown for the entire UMi sample (black), the metal-poor (blue), and metal-rich (red) populations.

cal ordering of the two populations is the same as other dSphs (e.g. Sculptor; Battaglia et al. 2008) despite only imposing a prior on $\sigma_{v}$. Although the metallicity separation is less than found in other dSphs, we will refer to the two stellar populations as the metal-rich and metal-poor populations. While the metallicity distributions overlap, the two stellar populations have distinct velocity, metallicity, and spatial distributions.

We show the spatial distribution of stars in each population in Fig. 5. Interestingly, the metal-rich population is significantly more flattened $(\epsilon=0.75 \pm 0.03)$ than the metal-poor population $(\epsilon=$ $\left.0.33_{-0.12}^{+0.09}\right)$ and UMi in general $(\epsilon=0.55 \pm 0.01)$. Both populations have major axes of similar length. In our standard analysis, we fixed the position angle. We have explored varying the position angle for each component and find that the difference in position angle between the two populations is small, $\Delta \theta<5^{\circ}$. The visual offset in Fig. 5 is due to the spectroscopic selection. While we account for the spectroscopic selection function, it is possible that there is a remaining bias. In Section 4.3, we examine and confirm the flattened metal-rich component with an independent UMi spectroscopic data set.

In Fig. 6, we examine the radial dependence of the average metallicity and metallicity dispersion of both populations. The two populations are clearly offset in mean metallicity in all radial bins. The difference in metallicity between the two populations is less than the differences observed in other dSphs (e.g. Fornax, Sculptor). The metallicity dispersion is larger in the metal-poor population compared to the metal-rich population.

Fig. 7 shows the radial dependence of $\sigma_{v}$ of the metal-rich and metal-poor populations. The metal-rich $\sigma_{v}$ is constant with radius, whereas the metal-poor $\sigma$ has hints that it deceases from $\approx 13 \mathrm{~km} \mathrm{~s}^{-1}$ at the centre to $\approx 10 \mathrm{~km} \mathrm{~s}^{-1}$ at large radii. For comparison, we also include the binned single-component velocity dispersion (black). Decreasing velocity dispersion profiles for subcomponents is not unusual in a dSph. They have been observed in the Fornax metalpoor population (Amorisco \& Evans 2012b) and in the Sculptor metal-rich population (Battaglia et al. 2008).

To test whether our assumption of a constant velocity dispersion affects the identification of stars within either chemodynamic population, we explore a model with a radial dependence. The 
velocity dispersion functional form we use is $\sigma_{v}\left(R_{\mathrm{e}}\right)=\sigma_{\mathrm{o}}(1+$ $\left.R_{\mathrm{e}} / R_{\sigma}\right)^{\alpha}$ (we use the same ellipticity for the spatial distribution and dispersion function). This model has two additional parameters: a radial scale, $R_{\sigma}$, and a power-law slope, $\alpha$. The priors for each parameter are $-2<\log _{10}\left(R_{\sigma} / 1 \mathrm{kpc}\right)<1$ and $-5<\alpha<5$. Overall, the inferred functional forms for the metal-rich and metal-poor are consistent with the binned profiles; the metal-rich population is constant with radius, whereas the metal-poor population decreases with radius from $\approx 13$ to $\approx 10 \mathrm{~km} \mathrm{~s}^{-1}$. The posterior distribution for velocity dispersion parameters is degenerates with one another and non-kinematic parameters change little compared to the constant velocity dispersion model. We find little change in the assignment of stars to either population. The net absolute change $\left(\sum \Delta\left|p_{\sigma\left(R_{\mathrm{e}}\right)}-p_{\text {const }}\right|\right)$ is $\sim 24$ and the maximum absolute change of an individual star is small $\left(\Delta p_{i}=0.16\right)$. The mean and median differences of the membership are both 0.03 and the standard deviation is 0.03 . Assuming a constant velocity dispersion model does not affect the identification of two populations or parameter inference.

Our UMi sample is built from three different observing epochs with very different target selection criteria (a combination of different spatial regions and a different photometric input catalogues). To explore whether the inhomogeneous target selection is driving our inference of the chemodynamic populations, we apply the same analysis on three subsets. Each subset excludes one of the three epochs and the subset excluding the 2009, 2010, and 2012 data contains 435, 554, and 302 stars, respectively. Applying the same analysis to each subset, we continue to identify two chemodynamic populations with high significance $(\ln B=23.57,15.18,25.14)$. Our inhomogeneous target selection does not affect the inference of two chemodynamic populations.

\subsection{Analysis with MMT/Hechocelle data}

As a cross-check, we search for and find two chemodynamic populations with the independent UMi spectroscopic survey from MMT/Hectochelle observations (Spencer et al. 2018). We first apply a similar mixture model to determine UMi membership and emphasize again that Gaia proper motions significantly improve dSph and foreground separation. The MMT sample contains brighter stars in general than the Keck sample and all but one star is in the Gaia DR2 catalogue. We select candidate UMi stars with a rough CMD selection with Gaia photometry $\left(G_{\mathrm{BP}}-G_{\mathrm{RP}}\right.$ versus $\left.G\right)$ based on a slightly expanded selection in fig. 3 of Gaia Collaboration (2018b). Using proper motion, velocity, metallicity, and spatial positions, we identify 413 stars as UMi members $\left(p_{\mathrm{dSph}}>0.95\right)$. Similar to the Keck/DEIMOS data set, very few stars have intermediate membership; only five stars have a membership in the range 0.01 $<p_{\mathrm{dSph}}<0.95$.

The overall UMi properties ${ }^{7}$ we find in this data set are similar to the Keck/DEIMOS data set: $\bar{v}=-246.9 \pm 0.4 \mathrm{~km} \mathrm{~s}^{-1}, \sigma_{v}=8.6 \pm$ $0.3 \mathrm{~km} \mathrm{~s}^{-1}, \overline{[\mathrm{Fe} / \mathrm{H}]}=-2.02 \pm 0.02, \sigma_{[\mathrm{Fe} / \mathrm{H}]}=0.37 \pm 0.02, r_{\mathrm{p}}=$ $450_{-30}^{+33} \mathrm{pc}, \epsilon=0.56 \pm 0.03$, and $R_{\mathrm{h}}=297 \pm 15 \mathrm{pc}$. We find a $\sigma_{v}$ value that is $\approx 0.6 \mathrm{~km} \mathrm{~s}^{-1}$ larger than Spencer et al. (2018). This difference is likely due to our use of Gaia DR2 astrometry and the different membership methods (mixture model versus $\sigma$ clipping).

\footnotetext{
${ }^{7}$ The mean velocity and metallicity we find for the MMT data are offset from the Keck value by $\approx 2.3 \mathrm{~km} \mathrm{~s}^{-1}$ and $\approx 0.13$, respectively. These are similar to the offsets found between common stars in Section 2.3.
}

Table 5. UMi membership of MMT/Hectochelle sample (Spencer et al 2018). Column (1): Gaia DR2 Source ID; column (2): membership in dSph MW model ( $\left.p_{\mathrm{dSph}}\right)$; column (3): membership in metal-rich population ( $\left.p_{\mathrm{MR}}\right)$; column (4): comments. CMD = star outside of colour-magnitude selection box and excluded from analysis. Gaia $=$ non-zero parallax and/or large proper motion and considered MW star. This table is available in its entirety in the electronic edition of the journal. A portion is reproduced here to provide guidance on form and content.

\begin{tabular}{lccc}
\hline Gaia DR2 Source ID & $p_{\mathrm{dSph}}$ & $p_{\mathrm{MR}}$ & Comments \\
\hline$(1)$ & $(2)$ & $(3)$ & $(4)$ \\
1645329064029028992 & 0.00 & - & CMD; Gaia \\
1645332259484700800 & 1.00 & 0.13 & \\
1645337580948516736 & 0.00 & - & Gaia \\
1645337958905643648 & 1.00 & 0.19 & \\
1645338130704328064 & 1.00 & 0.61 & \\
1645339024057541120 & 1.00 & 0.77 & \\
\hline
\end{tabular}

We find statistically significant evidence for two chemodynamic populations in the MMT/Hectochelle data set $(\ln B=17.94)$. The overall results are similar to the Keck/DEIMOS sample; the first population is centrally concentrated, dynamically cold, and metalrich, whereas the secondary component is more extended, kinematically hot, and metal-poor. The properties of the two populations are summarized in the last two columns of Table 4. In Table 5, we list our dSph and metal-rich membership for stars in the MMT sample. With the MMT data, we confirm the metal-rich population is aspherical $(\epsilon=0.64 \pm 0.04)$, while the extended population is more spherical $\left(\epsilon=0.21_{-0.14}^{+0.16}\right.$, constrained to be $\epsilon<0.38$ at the 90 per cent confidence interval). We note that the two populations are closer in velocity dispersion $\left(\Delta \sigma_{v} \sim 4.4 \mathrm{~km} \mathrm{~s}^{-1}\right)$ than our results in the Keck data set $\left(\Delta \sigma_{v} \sim 6.6 \mathrm{~km} \mathrm{~s}^{-1}\right)$. The MMT metal-poor $\sigma_{v}$ is consistent within errors with the Keck measurement, whereas the metal-rich $\sigma_{v}$ is larger and disagrees at $\sim 2 \sigma$. The metal-rich component is more metal-rich than the Keck results; this may be due to offset found between the samples based on repeated measurements (see Section 2.3 and Fig. 2). The MMT metal-poor population is more extended but more uncertain compared to the Keck results (due to the overall lower number of stars). We find an overall smaller fraction of stars in the metal-poor population $(f=0.24$ versus $f=$ $0.46)$. The differences between the inferred properties may be based on differences in the target selection in the two data sets.

In Fig. 8, we show the binned $\sigma_{v}$ profiles with the MMT data. The metal-poor $\sigma_{v}$ declines from $\approx 15$ to $\approx 10 \mathrm{~km} \mathrm{~s}^{-1}$ and the metal-rich population is constant with radius. This confirms the $\sigma_{v}$ profile of the metal-poor population that was seen in the Keck data.

The results from the MMT sample independently confirm the two chemodynamical populations in UMi. Moreover, it confirms two interesting features in UMi: a flattened metal-rich population with an almost spherical metal-poor component and a declining $\sigma_{v}$ profile for the metal-poor population. For the following analysis, we will analyse both UMi data sets independently. We opt not to combine the data sets due to the observed offsets in velocity and metallicity and the different methodologies for velocity and metallicity measurements.

\subsection{Search for rotation}

The flattened nature of the metal-rich population is reminiscent of a disc galaxy and a natural question is whether it is rotating. We first search for rotation with a simple test, splitting the sample in half based on bisecting lines at different position angles and 


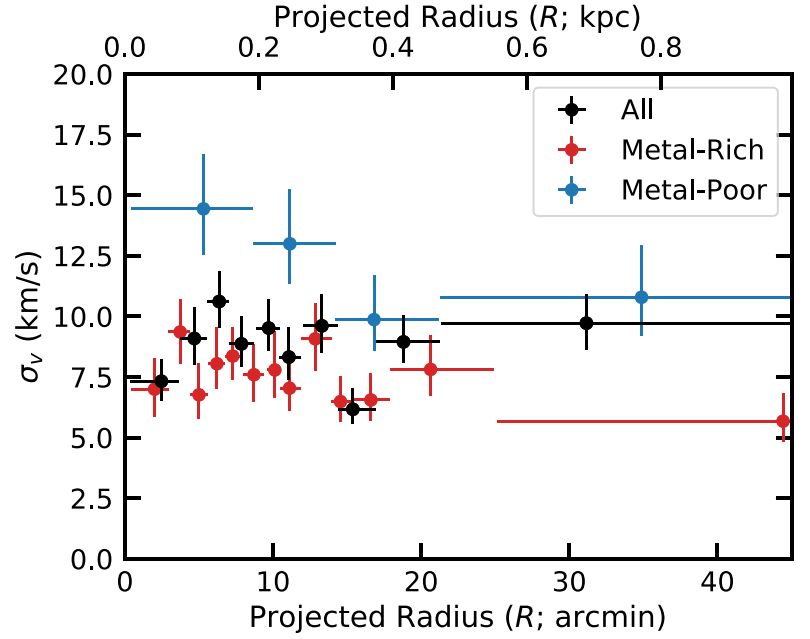

Figure 8. Same as Fig. 7 except with the MMT/Hectochelle data set (Spencer et al. 2018). The metal-poor components contain 25 stars per bin, while the other components contain 40 stars.

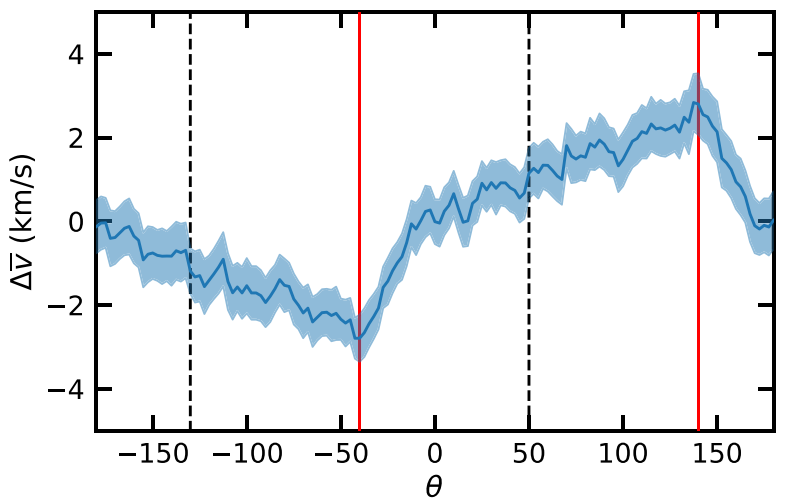

Figure 9. Difference between the mean average velocity for each half of the sample based on bisecting lines for different rotation axes for the MMT/Hectochelle data set. The angles $\theta=-130^{\circ}, 50^{\circ}$ correspond to the photometric major axis (dashed black lines), and the angles $\theta=-40^{\circ}, 140^{\circ}$ correspond to the photometric minor axis (solid red lines). There are hints of rotation along the minor axis (i.e. prolate rotation).

computing the difference between the mean velocity in each half. The entire Keck RGB data set and the Keck metal-rich population show little to no signal, whereas the Keck metal-poor population has an amplitude of $\sim 2 \mathrm{~km} \mathrm{~s}^{-1}$. In contrast, we observe a signal in the entire MMT data set, the MMT metal-rich population, and the MMT metal-poor population with amplitudes of $\sim 3, \sim 6$, and $\sim 2 \mathrm{~km} \mathrm{~s}^{-1}$, respectively. In all cases with a signal, including the Keck metal-poor population, the peak amplitude occurs near the photometric minor axis corresponding to prolate rotation $\left(\theta=-40^{\circ}\right.$, $140^{\circ}$ correspond to the photometric minor axis). ${ }^{8}$ We show the results of this test for the MMT data set in Fig. 9. We note that this is one of the better examples of rotation in the subsets examined.

To further quantify any potential rotation signal, we explore a rotation model that is constant with radius. We add the rotation term to the relative velocity: $v_{\text {rel }}=\bar{v}+A_{\text {rot }} \cos \left(\theta-\theta_{\text {rot }}\right.$ ) (Wheeler

${ }^{8}$ The Muñoz et al. (2005) data set also shows evidence for rotation with an amplitude of $\sim 5 \mathrm{~km} \mathrm{~s}^{-1}$ that peaks at a similar position angle. et al. 2017). This includes two additional parameters, an amplitude, $A_{\text {rot }}$, and a rotation angle, $\theta_{\text {rot }}$. In the Keck RGB data set, the rotation amplitudes we derive are consistent with zero and we find upper limits of $A_{\text {rot }}<2.0,<4.0$, and $<2.2 \mathrm{~km} \mathrm{~s}^{-1} \mathrm{kpc}^{-1}$ (upper limits are at 95 per cent confidence) for the metalrich population, metal-poor population, and the entire sample, respectively. ${ }^{9}$ In the MMT data set, we measure rotation amplitudes and rotation axes of $A_{\text {rot }}=1.9 \pm 0.9 \mathrm{~km} \mathrm{~s}^{-1} \mathrm{kpc}^{-1}$ and $\theta_{\text {rot }}=$ $156_{-17^{\circ}}^{\circ}, 4^{\circ}, A_{\text {rot }}=3.6 \pm 2.0 \mathrm{~km} \mathrm{~s}^{-1} \mathrm{kpc}^{-1}$ and $\theta_{\text {rot }}=164_{-33^{\circ}}^{\circ}$, and $A_{\text {rot }}=1.8 \pm 0.8 \mathrm{~km} \mathrm{~s}^{-1} \mathrm{kpc}^{-1}$ and $\theta_{\text {rot }}=156_{-19^{\circ}}^{\circ}$ for the metalrich population, metal-poor population, and the entire sample, respectively. The rotation angle agrees with the simple rotation test and is suggestive of prolate rotation.

We set upper limits with the Keck data set (i.e. posterior of $A_{\text {rot }}$ in the Keck data set is generally maximized at zero) and infer rotation with the MMT data set (i.e. the $A_{\text {rot }}$ posterior peaks at a non-zero value). The different conclusion could be due to the larger spatial extent of the MMT data set and/or due to the differences in the velocity errors between the two instruments. The median velocity error of the Keck and MMT data sets is 2.6 and $0.7 \mathrm{~km} \mathrm{~s}^{-1}$, respectively. Given these differences it is more likely that low levels of rotation could be observed with the MMT data set despite the larger Keck data set.

Interestingly, the rotation axis inferred from the MMT data is similar to the velocity gradient induced by the perspective motion. Based on the proper motion measured from Gaia DR2, the maximum magnitude of perspective motion effect is $\pm 0.2 \mathrm{~km} \mathrm{~s}^{-1}$. This effect is already included in all of our modelling and is much smaller than the rotation amplitudes we infer (see Section 3.2). Rotation is not favoured in any of the data sets examined compared to the non-rotating model. Additional extended data sets, especially along the minor axis, are required to confirm or refute the hint of prolate rotation.

Prolate rotation has been previously observed in two dwarf galaxies in the local: the M31 satellite dSph, And II (Ho et al. 2012) and the isolated transition-type dwarf galaxy, Phoenix (Kacharov et al. 2017). In both cases, prolate rotation has been argued to be evidence for a recent merger. For example, the peculiar kinematics of And II may be due to a merger at $z \sim 1.75$ (Amorisco, Evans \& van de Ven 2014; Lokas et al. 2014; del Pino et al. 2017b; Fouquet et al. 2017). In Phoenix, the spatial distribution of the young stars is aligned with the rotation axis/minor axis that is further evidence for a recent merger (Kacharov et al. 2017). Prolate rotation has been observed for a subset of galaxies in the Illustris hydrodynamic simulation and the prolate rotation generally emerges from late mergers with radial orbits (Ebrová \& Łokas 2017). The hints of prolate rotation in UMi may be evidence for a recent merger.

\subsection{Exploring additional populations}

A natural question is whether UMi contains additional chemodynamic populations, similar to Fornax (Amorisco \& Evans 2012b) and Carina (Kordopatis et al. 2016). With our formalism it is straightforward to extend our analysis to an additional component. We explore three different priors to disentangle populations: $\sigma_{v, 1}$ $<\sigma_{v, 2}<\sigma_{v, 3},[\mathrm{Fe} / \mathrm{H}]_{1}<[\mathrm{Fe} / \mathrm{H}]_{2}<[\mathrm{Fe} / \mathrm{H}]_{3}$, and the two first two

${ }^{9}$ We have explored this test by adding rotation to one component at a time and both components simultaneously with similar results. 
priors combined. For the fractions parameters, we use $f_{x}$ and $f_{y}$ as free parameters with the prior range $0<f_{x, y}<1$. The transformations from these parameters to the population fraction parameters are $f_{1}=$ $1-f_{x}, f_{2}=f_{x}\left(1-f_{y}\right)$, and $f_{3}=f_{x} f_{y}$.

With the Keck data, we find weak to moderate evidence in favour of the three population model when compared to the two population model ( $\ln B=3.09,3.33$, and 1.61 for the velocity dispersion, metallicity, and combined prior, respectively). The different priors do not affect the posterior distributions of the velocity dispersion, metallicity, and half-light radii of the three components and we observe the expected chemodynamic ordering in all three cases. We will refer to the three components here as ' 1 ', ' 2 ', and ' 3 ' and ordering them from highest to lowest metallicity. With the velocity dispersion prior, for the first population we find $\sigma_{v, 1}=$ $4.7_{-0.9}^{+0.7} \mathrm{~km} \mathrm{~s}^{-1}, \overline{[\mathrm{Fe} / \mathrm{H}]}=-2.00_{-0.04}^{+0.04}, \sigma_{[\mathrm{Fe} / \mathrm{H}], 1}=0.25_{-0.03}^{+0.03}, \epsilon_{1}=$ $0.75_{-0.03}^{+0.03}$, and $R_{\mathrm{h}, 1}=214_{-17}^{+17} \mathrm{pc}$. For the second population we find $\sigma_{v, 2}=9.9_{-1.4}^{+1.1} \mathrm{~km} \mathrm{~s}^{-1}, \overline{[\mathrm{Fe} / \mathrm{H}]}_{2}=-2.29_{-0.04}^{+0.05}, \sigma_{[\mathrm{Fe} / \mathrm{H}], 2}=0.09_{-0.05}^{+0.34}$, $\epsilon_{2}=0.54_{-0.25}^{+0.13}$, and $R_{\mathrm{h}, 2}=347_{-57}^{+73} \mathrm{pc}$. For the third population we find $\sigma_{v, 3}=11.8_{-0.9}^{+1.0} \mathrm{~km} \mathrm{~s}^{-1}, \frac{-57 \mathrm{Fe} / \mathrm{H}]_{3}}{\left[R_{\mathrm{h}, 3}\right.}=-2.31_{-0.07}^{+0.07}, \sigma_{[\mathrm{Fe} / \mathrm{H}], 3}=$ $0.42_{-0.31}^{+0.06}, \epsilon_{3}=0.27_{-0.16}^{+0.20}$, and $R_{\mathrm{h}, 3}=377_{-58}^{+71} \mathrm{pc}$. The metallicity dispersion posterior is multimodal in the second and third populations with peaks at $\sigma_{[\mathrm{Fe} / \mathrm{H}]} \sim 0.10$ and $\sim 0.45$. The fraction of stars in each component is $f_{1}=0.46_{-0.09}^{+0.08}, f_{2}=0.26_{-0.06}^{+0.07}$, and $f_{3}=0.27_{-0.07}^{+0.08}$. The most metal-rich component in both the twoand three-population modelling has similar properties. The ' 2 ' and ' 3 ' populations are similar to a splitting of the original 'metal-poor' population. Comparing with star-by-star membership confirms this picture, stars originally in the 'metal-rich' component are more likely to be in population ' 1 ', while 'metal-poor' stars are more likely to be in populations ' 2 ' or ' 3 '.

With the MMT data, we find inconclusive to moderate evidence in favour of the three-population model compared to the two-population model $(\ln B=1.65,2.68$, and 0.93 for the $\sigma$, metallicity, and combined prior, respectively). In contrast to the Keck data, we find inconsistent results with the different priors analysing the MMT data. Chemodynamic ordering is not observed between the three components with any of priors and is different depending on the prior used. With all three priors the most metalpoor component (' 3 ') has similar properties and always has a larger spatial scale and velocity dispersion. With the metallicity prior $\left(\overline{[\mathrm{Fe} / \mathrm{H}}_{1}<{\overline{[\mathrm{Fe} / \mathrm{H}]_{3}}}_{3}<\overline{[\mathrm{Fe} / \mathrm{H}]_{3}}\right.$ ), we find $\sigma_{v, 3}>\sigma_{v, 1}>\sigma_{v, 2}$ and $R_{\mathrm{h}, 3}>R_{\mathrm{h}, 2}>R_{\mathrm{h}, 1}$. With the velocity dispersion prior $\left(\sigma_{v, 3}>\right.$ $\sigma_{v, 2}>\sigma_{v, 1}$ ), we find $\overline{[\mathrm{Fe} / \mathrm{H}]_{1}} \approx \overline{[\mathrm{Fe} / \mathrm{H}]_{2}}<\overline{[\mathrm{Fe} / \mathrm{H}]_{3}}$ and $R_{\mathrm{h}, 3}>$ $R_{\mathrm{h}, 1}>R_{\mathrm{h}, 2}$. With the combined prior we find $R_{\mathrm{h}, 3}>R_{\mathrm{h}, 1} \approx R_{\mathrm{h}, 2}$. The three-population modelling with different priors does not have consistent results with the MMT data.

Our three-component results for the Keck data set are consistent across the three different priors we used and the Bayesian evidence favours three components moderately. This moderate evidence must be interpreted with care. Even if more data would increase the evidence for three components, it would not necessarily argue for three distinct chemodynamic populations. The bimodal posterior of $\sigma_{[\mathrm{Fe} / \mathrm{H}]}$ seems likely to be driven by the non-Gaussian distribution of $[\mathrm{Fe} / \mathrm{H}]$ for the metal poor population. Chemical evolution models are known to produce non-Gaussian metallicity distributions (e.g. Kirby et al. 2011), hence the conservative interpretation is that we have two distinct chemodynamic populations with more complex metallicity distributions. A straightforward test is to examine a two-population model where the metallicity distribution of the metal-poor component contains two Gaussians instead of one. With this model we find that mean metallicities are equivalent

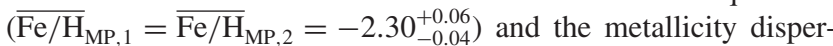

sion parameter mirror the results with the three-population model $\left(\sigma_{[\mathrm{Fe} / \mathrm{H}], \mathrm{MP}, 1}=0.09_{-0.04}^{+0.04}\right.$ and $\left.\sigma_{[\mathrm{Fe} / \mathrm{H}], \mathrm{MP}, 2}=0.48_{-0.04}^{+0.04}\right)$. This model is a better fit compared to the three-component models $(\ln B=2.83$ compared to the best-fitting three-component model). There is some evidence that the axis ratios could be different for the second and third components, but this could be an indication that the luminosity profile for the metal-poor component deviates from the assumed Plummer profile. As points of comparison, we note that the two $\mathrm{dSphs}$ with three reported chemodynamical populations, Carina and Fornax both have larger stellar masses and have more extended star formation histories than UMi (Weisz et al. 2014). In addition, the reported chemodynamic populations have larger metallicity differences than our inferences for the second and third components. Given the reasons above, we conclude that the evidence for a distinct third chemodynamic population in UMi is weak.

\section{SIMPLE ESTIMATORS OF MASS SLOPE}

Although the overall mass profile is degenerate with the stellar anisotropy, the mass within the stellar half-light radius, $M_{\mathrm{h}}=M\left(r_{\mathrm{h}}\right)$, is well determined for spherical systems (Walker et al. 2009; Wolf et al. 2010). The difference in mass at the half-light radii of each chemodynamic population provides an estimate for the mass slope: $\Gamma=\Delta M_{\mathrm{h}} / \Delta r_{\mathrm{h}}$ (Walker \& Peñarrubia 2011). To accommodate the assumption of spherical symmetry, we use the geometric mean of the major and minor axes as a 'spherical' half-light radius (azimuthally averaged half-light radius, $R_{\mathrm{h}}=r_{\mathrm{p}} \sqrt{q}$ ). The modified mass-slope estimator is

$\Gamma=1+\frac{\ln \left[\sigma_{v, 1}^{2} / \sigma_{v, 2}^{2}\right]}{\ln \left[\left(r_{\mathrm{p}, 1} \sqrt{q_{1}}\right) /\left(r_{\mathrm{p}, 2} \sqrt{q_{2}}\right)\right]}$.

The benefit of this method is that no additional dynamical modelling is required; only quantities directly measured in the two-component analysis are used. However, this estimator may not be valid for UMi. The potential stellar rotation, extreme ellipticity, and non-constant $\sigma_{v}$ invalidate several of the estimator's assumptions. While we have accounted for the ellipticity, these estimators have not been tested at high ellipticity. Note that $\Gamma$ is not a direct measurement of the inner density profile (at $r=0$ ) but a measurement of the average slope of the mass profile between $R_{\mathrm{h}}$ of the two populations. For a dark matter density profile scaling as $\rho_{\mathrm{DM}} \propto r^{-\gamma_{\mathrm{DM}}}$ in the central regions, $\Gamma$ places an upper limit on the density slope as $\gamma_{\mathrm{DM}}<3-$ $\Gamma$ (Walker \& Peñarrubia 2011). For a Navarro-Frenk-White (NFW; or 'cuspy') profile, $\gamma_{\mathrm{DM}}=1$ and $\Gamma=2$. For a constant ('cored') density profile, $\gamma_{\mathrm{DM}}=0$ and $\Gamma=3$.

With these caveats, we measure $\Gamma=4.2_{-0.9}^{+1.6}$ in the radial range of $\approx 200-400$ pc with the Keck data set. We display the posterior distributions of $\Gamma$ in Fig. 10. This disfavours a 'cuspy' NFW halo $(\Gamma=2)$ at greater than $2 \sigma$ but the majority of the posterior has $\Gamma>3$, implying a unphysical density that increases with radius (assuming $\rho_{\mathrm{DM}} \propto r^{-\gamma_{\mathrm{DM}}}$ ). This tendency to favour large $\Gamma$ is due to the large difference in $\sigma_{v}$ but smaller relative difference in $R_{\mathrm{h}}$. These quantities could misestimated due to the non-constant $\sigma_{v}$ or the large ellipticity. For comparison we find $\Gamma=2.3_{-0.5}^{+0.8}$ with the MMT data set, which favours a 'cuspy' halo but it is not precise enough to distinguish between 'cored' or 'cuspy' haloes. The $\Gamma$ posterior distributions of the Keck and MMT data sets overlap but favour different interpretations of the inner dark matter slope. The disagreement between the two data sets is caused by the difference in metal-poor population $R_{\mathrm{h}}$ and the difference in metalrich population $\sigma_{v}$. Simply increasing the Keck metal-poor $R_{\mathrm{h}}$ by 50 per cent results in much better $\Gamma$ agreement between the two 


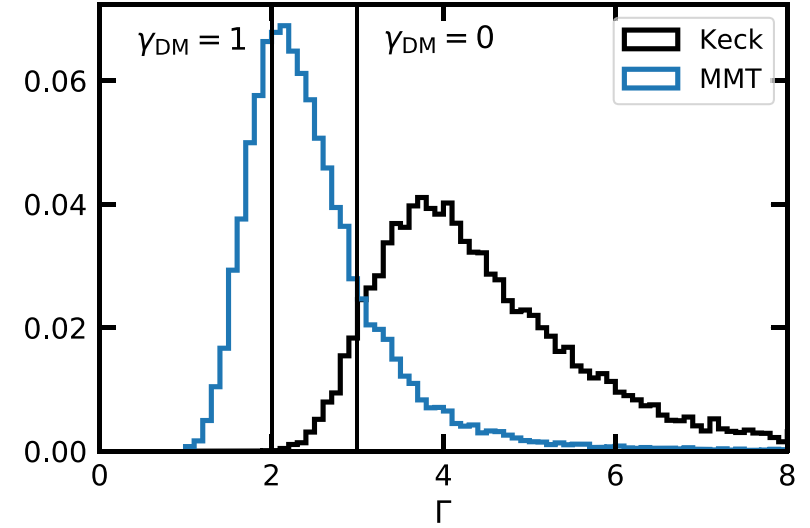

Figure 10. Posterior distributions of the mass slope $(\Gamma)$ for the Keck/DEIMOS (black) and MMT/Hectochelle (blue) data sets. The two lines show where 'cuspy' (left; $\Gamma=2$ ) and 'cored' (right; $\Gamma=3$ ) haloes lie in the $\Gamma$ distribution. The Keck data set favours very large $\Gamma$ that may indicate the breakdown of the simple estimator (i.e. non-constant velocity dispersion in the metal-poor component or the large ellipticity of the metalrich component). The MMT data set favours a 'cuspy' distribution but does not exclude a 'cored' dark matter halo.

data sets. A more extended data set will be key to determining the inner slope of the dark matter density profile.

The half-light mass estimators that $\Gamma$ is based on have been verified to be robust in a wide range of systems and $\mathrm{N}$-body simulations (Walker \& Peñarrubia 2011; Kowalczyk et al. 2013; Laporte, Walker \& Peñarrubia 2013; Lyskova et al. 2015; Campbell et al. 2017; González-Samaniego et al. 2017; Errani, Peñarrubia \& Walker 2018). There has been a healthy discussion about the robustness of the $\Gamma$ estimator (Walker \& Peñarrubia 2011; Kowalczyk et al. 2013; Laporte et al. 2013; Genina et al. 2018). More recent results on the viewing angle suggest that there may be biases in the estimator (Walker \& Peñarrubia 2011; Laporte et al. 2013).

\section{CHEMODYNAMIC POPULATIONS THROUGHOUT THE LOCAL GROUP}

Multiple distinct global chemodynamic populations ${ }^{10}$ have been found with high significance in several dSphs. Chemodynamic populations are structured such that the inner central concentrated population is metal-rich and kinematically cold, while the outer extended population is metal-poor and kinematically hot. The two populations that we have uncovered in UMi follow these same trends. Chemodynamical populations have encouraged a vast amount of dynamical modelling analysis (e.g. Battaglia et al. 2008; Agnello \& Evans 2012; Amorisco \& Evans 2012a; Strigari et al. 2017; Hayashi et al. 2018).

Multiple populations have been detected in Carina ${ }^{11}$ (Fabrizio et al. 2016; Kordopatis et al. 2016; Hayashi et al. 2018), Fornax

\footnotetext{
${ }^{10}$ Localized kinematic or chemodynamical substructure has been seen or claimed in multiple dSphs. However, detections are not always consistent between different data sets and methods (Kleyna et al. 2003, 2004; Walker et al. 2006; Battaglia et al. 2011; Fabrizio et al. 2011; Amorisco et al. 2014; Pace et al. 2014; del Pino et al. 2017a,b; Cicuéndez \& Battaglia 2018; Kim et al. 2019; Lora et al. 2019).

${ }^{11}$ The intermediate and metal-rich populations in Carina are an exception to the general chemodynamic ordering trend. The intermediate-metallicity population is the most compact and kinematically cold (Kordopatis et al. 2016). It is unclear why in Carina the populations are mixed in this manner
}

(Battaglia et al. 2006; Walker \& Peñarrubia 2011; Amorisco \& Evans 2012b; Amorisco et al. 2013; Hendricks et al. 2014), Sculptor (Tolstoy et al. 2004; Battaglia et al. 2008; Walker \& Peñarrubia 2011; Zhu et al. 2016), Sagittarius ${ }^{12}$ (Majewski et al. 2013), and UMi (this work). There are claims or lower significance detections in several other dSphs. For example, Ibata et al. (2006) claim a detection in Canes Venatici I. However, larger data sets do not confirm this feature (Simon \& Geha 2007; Ural et al. 2010). Similarly, Koposov et al. (2011) find that Boötes I favours a twocomponent model in kinematics, while previous data sets did not find this (Muñoz et al. 2006; Martin et al. 2007). There is tentative evidence in the local field isolated dSphs Cetus (Taibi et al. 2018) and Tucana (Taibi et al. 2020) and hints in Leo II (Spencer et al. 2017) and Sextans (Battaglia et al. 2011) that may be confirmed with larger sample sizes.

While the chemodynamical populations that we have uncovered in UMi are similar to the standard chemodynamic ordering, there are some interesting differences. First, the metallicity distributions in UMi are closer than in other dSphs. This could be due to a merger of two similar-sized galaxies or there was little gas enrichment before the formation of the second component. Second, the UMi metalrich population is flattened and significantly more elongated than the more spherical metal-poor population. UMi is more elongated ( $\epsilon=0.56)$ than the other classical $(0.07<\epsilon<0.45)$ satellites (Muñoz et al. 2018b). Chemodynamic analyses of other dSphs have found similar ellipticities for each population (Carina, Fornax, and Sculptor; Kordopatis et al. 2016; Zhu et al. 2016; Hayashi et al. 2018). Third, the metal-poor $\sigma_{v}$ decreases $\approx 5$ to $\approx 10 \mathrm{~km} \mathrm{~s}^{-1}$ beyond $\approx 300 \mathrm{pc}$. Declining $\sigma_{v}$ have been observed in the Fornax metal-poor population (Amorisco \& Evans 2012b) and the Sculptor metal-rich population (Battaglia et al. 2008) chemodynamic analysis. It is interesting that as a whole the classical dSphs have constant $\sigma_{v}$ with radius (Walker et al. 2007), whereas some subpopulations are observed to deviate from this trend. Each of these characteristics may be related to the formation mechanism of the two populations.

Several formation scenarios for multiple populations have been proposed, including mergers (Amorisco \& Evans 2012b; del Pino, Aparicio \& Hidalgo 2015; Genina et al. 2019), supernova feedback (Salvadori, Ferrara \& Schneider 2008; Revaz et al. 2009), tidal interactions (Pasetto et al. 2011), interactions with a gaseous cosmic filament (Genina et al. 2019), or compression of gas during infall (Genina et al. 2019). In addition, they are seen in isolated hydrodynamical simulations (Revaz \& Jablonka 2018). In the merger scenario, the spatial separation is due to metal-poor stars migrating to outer orbits with the metal-rich population forming in situ after the merger (Genina et al. 2019). Based on Gaia DR2 proper motions, the infall time of UMi is 10.7 Gyr (Fillingham et al. 2019) and is early enough that the metal-rich population could have formed due to gas compression from ram pressure stripping during MW infall. Large differences between the shapes of two populations are not seen in simulated dSph satellites (Genina et al. 2018). In field dwarfs, the metal-poor populations are more spherical than the metal-rich populations. However, this difference

relative to other dSphs. The star formation in Carina is episodic and so distinct and well separated that they can be clearly seen without any special modelling (Hurley-Keller, Mateo \& Nemec 1998; de Boer et al. 2014). Furthermore, Walker \& Peñarrubia (2011) have a null detection in Carina.

${ }^{12}$ Chemodynamic trends have also been observed in the leading and trailing arms of the Sagittarius stellar stream (Gibbons, Belokurov \& Evans 2017). 
is smaller in satellites, likely due to tidal stripping (Genina et al. 2018, 2019). At the lowest stellar masses, Genina et al. (2019) find that most multiple populations in $\mathrm{dSphs}$ are primarily formed due to mergers. Observations of prolate rotation may be evidence for past mergers (Amorisco et al. 2014; Kacharov et al. 2017) and the two populations in UMi could be evidence for a late-time merger. We note that Genina et al. (2019) conclude that spatial and kinematic information is insufficient to determine the formation mechanism but metallicities and star formation histories can provide clues on their origin. The multiple populations in UMi could have formed due to mergers or gas interactions but it is not yet conclusive which mechanism is responsible. Future star formation history constraints combined with metallicity distribution functions will assist in disambiguating between these scenarios.

\section{DARK MATTER ANNIHILATION AND DECAY RATES OF URSA MINOR}

The close proximity and high dark matter content of the MW dSphs make them ideal candidates to search for signatures of dark matter annihilation or decay (Baltz et al. 2000; Tyler 2002; Evans, Ferrer \& Sarkar 2004; Bergström \& Hooper 2006; Colafrancesco, Profumo \& Ullio 2007; Strigari et al. 2007a). The dark matter annihilation or decay flux from a dSph depends on the distribution of dark matter within the $\mathrm{dSph}$ (the astrophysical component) and properties of the dark matter particle such as the mass or cross-section (the particle physics component). For velocity independent models these components are separable and the astrophysics portion is referred to as the $J$ - and $D$-factor for annihilation and decay, respectively. The $J$ - and $D$-factors are

$$
J\left(\theta_{\max }\right)=\iint_{\operatorname{los}} \rho_{\mathrm{DM}}^{2}(l, \Omega) \mathrm{d} l \mathrm{~d} \Omega
$$

and

$D\left(\theta_{\max }\right)=\iint_{\operatorname{los}} \rho_{\mathrm{DM}}(l, \Omega) \mathrm{d} l \mathrm{~d} \Omega$.

The integrals are over the line-of-sight direction and a solid angle centred on the dwarf. $\theta_{\max }$ is the maximum angle probed. The standard methodology for determining $\rho_{\mathrm{DM}}$ for $\mathrm{dSphs}$ is with dynamical models based on the spherical Jeans equation (e.g. Strigari et al. 2007a; Walker et al. 2011; Bonnivard et al. 2015; Geringer-Sameth, Koushiappas \& Walker 2015; Pace \& Strigari 2019). For a spherically symmetric system in dynamical equilibrium the spherical Jeans equations reduce to a single differential equation (Binney \& Tremaine 2008):

$\frac{\mathrm{d} v \sigma_{\mathrm{r}}^{2}}{\mathrm{~d} r}+\frac{2}{r} \beta(r) v \sigma_{\mathrm{r}}^{2}+v \frac{G M(r)}{r^{2}}=0$,

where $v(r)$ is the 3D stellar density distribution, $M(r)$ is the mass distribution, $\sigma_{r}$ is the radial velocity dispersion, and $\beta$ is the stellar anisotropy, which is a function of the tangential and radial velocity dispersion, $\beta(r)=1-\sigma_{\mathrm{t}}^{2}(r) / \sigma_{\mathrm{r}}^{2}(r)$. To compare the theoretical velocity dispersion profiles to observed line-of-sight velocity data, we project the radial dispersion into the line-of-sight direction,

$\Sigma(R) \sigma_{\operatorname{los}}^{2}(R)=2 \int_{R}^{\infty} \mathrm{d} r\left(1-\beta(r) \frac{R^{2}}{r^{2}}\right) \frac{r v \sigma_{\mathrm{r}}^{2}(r)}{\sqrt{r^{2}-R^{2}}}$,

where $R$ is the projected radial distance, $\Sigma(R)$ is the projected tracer distribution (2D), and $\sigma_{\text {los }}^{2}$ is the velocity dispersion in the line-ofsight direction.
We assume a Plummer profile for the tracer profile (Plummer 1911). The 3D spherically symmetric analogue to the projected (2D) Plummer profile (equation 11) is

$\nu(r)=\frac{3}{4 \pi r_{\mathrm{p}}^{3}}\left[1+\left(r / r_{\mathrm{p}}\right)^{2}\right]^{-5 / 2}$.

To approximate spherical symmetry we use the spherically averaged half-light radii $\left(R_{\mathrm{h}}=r_{\mathrm{p}} \sqrt{q}\right)$. We assume that the mass profile is entirely dominated by the dark matter halo. We assume an Einasto dark matter halo profile (Einasto 1965; Navarro et al. 2004),

$\rho_{\text {Einasto }}(r)=\rho_{-2} \exp \left\{-\frac{2}{\alpha}\left[\left(\frac{r}{r_{-2}}\right)^{\alpha}-1\right]\right\}$,

where $r_{-2}, \rho_{-2}$, and $\alpha$ are the scale radius, scale density, and slope parameter, respectively. For the stellar anisotropy profile we use the generalized Baes \& van Hese (2007) profile,

$\beta(r)=\frac{\beta_{0}+\beta_{\infty}\left(r / r_{\beta}\right)^{\eta}}{1+\left(r / r_{\beta}\right)^{\eta}}$,

where $\beta_{0}, \beta_{\infty}, r_{\beta}$, and $\eta$ are parameters that correspond to the inner anisotropy, outer anisotropy, radial anisotropy scale, and transition slope, respectively.

We assume an unbinned likelihood (Strigari et al. 2008; GeringerSameth et al. 2015). We weight each star by its membership probability (equation 6).

To set the maximum size of the dark matter halo (for equation 13), we calculate the satellite's tidal radius, $r_{\mathrm{t}}=$ $d\left(M_{\mathrm{dSph}} / M_{\mathrm{MW}}(d)\left(2-\frac{\mathrm{d} \ln M_{\mathrm{MW}}}{\mathrm{d} \ln r}\right)\right)^{1 / 3}$ (Springel et al. 2008). We use the MW total mass profile from Eadie \& Harris (2016). We find $r_{\mathrm{t}}$ $\approx 5-6 \mathrm{kpc}$ for UMi. The majority of the $J$-factor comes from the inner region of the dark matter halo and small changes in $r_{\mathrm{t}}$ do not significantly impact $J$. We note that the UMi has one of the lowest pericentres of the classical satellites ( $r_{\text {peri }} \approx 30 \mathrm{kpc}$ ), and a more accurate $r_{\mathrm{t}}$ calculation should account for the full orbit of UMi (Fritz et al. 2018; Gaia Collaboration 2018b).

We examine two samples for each data set, the entire RGB sample and the metal-poor population. We do not dynamically model the metal-rich population or both populations simultaneously due to the large ellipticity in the metal-rich component. The metalpoor population is much more spherical and spherical Jeans may have some unknown systematics when modelling highly flattened systems. We model the RGB sample to provide a comparison sample that is selected in a similar manner to literature UMi $J$ factor measurements. Our spherical Jeans fits for the Keck data samples are shown in Fig. 11 and can adequately explain the stellar kinematics. For the $J$-factor $\left(\log _{10} J(\theta=0.5)=\log _{10} J\right)$, we derive $\log _{10} J=18.73_{-0.11}^{+0.17}$ and $18.47_{-0.09}^{+0.13}$ for the RGB samples for the Keck and MMT data sets, respectively. Our results for the metal-poor component are larger. We derive $\log _{10} J=19.08_{-0.13}^{+0.16}$ and $18.61_{-0.31}^{+0.31}$ for the metal-poor populations for the Keck and MMT data sets, respectively. Our $J$ - and $D$-factor results for additional angles are summarized in Table 6. Several recent literature measurements of the $J$-factor are $\log _{10} J=18.93_{-0.19}^{+0.27}$ (GeringerSameth et al. 2015), $\log _{10} J=18.8 \pm 0.19$ (Ackermann et al. 2014), $\log _{10} J=19.0 \pm 0.1$ (Bonnivard et al. 2015), $\log _{10} J=18.75 \pm 0.12$ (Pace \& Strigari 2019), and $\log _{10} J=18.75_{-0.13}^{+0.17}$ (Horigome et al. 2020). In general, our Keck results agree with recent values in the literature, however, our MMT results are smaller. 


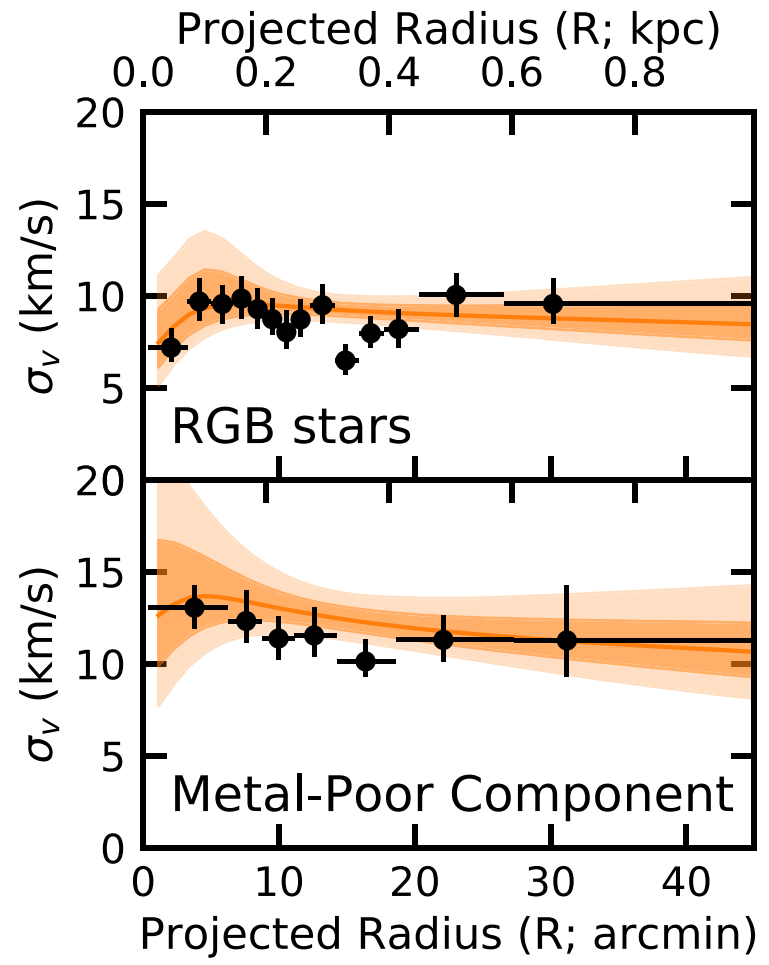

Figure 11. Binned velocity dispersion profiles (black points) overlaid with spherical Jeans fits (orange lines/bands) for the Keck/DEIMOS data set. The shaded bands show the $1 \sigma$ and $2 \sigma$ confidence intervals. The top (bottom) panel shows the RGB (metal-poor) stars. Note that the binned velocity dispersion profiles are for visualization only and the fit is done on a star-bystar basis.

The $J$-factor has a simple scaling based on the velocity dispersion, distance, and stellar size: $J \propto \sigma_{v}^{4} / d^{2} / r_{1 / 2}$ (Pace \& Strigari 2019). ${ }^{13}$ The differences in these inputs can explain a large portion of these differences. Generally, our assumed distance is further away, the stellar size larger, and the velocity dispersion smaller than other studies that will all decrease our measurement compared to the literature measurements. As a direct comparison we have repeated the analysis in Pace \& Strigari (2019) for the Keck and MMT data set. Pace \& Strigari (2019) assumed an NFW dark matter density profile, a constant stellar anisotropy, used the stellar parameters from Muñoz et al. (2018b), and varied the distance with a Gaussian prior. We derive $\log _{10} J=18.58 \pm 0.12$ and $18.64 \pm 0.11$ with the Keck and MMT data sets, respectively, with these modelling assumptions compared to $\log _{10} J=18.75 \pm 0.12$. The UMi data $^{14}$ utilized by Pace \& Strigari (2019) contained 311 stars with $\sigma_{v}=9.3 \pm 0.4 \mathrm{~km} \mathrm{~s}^{-1}$ compared to $\sigma_{v}=8.6 \mathrm{~km} \mathrm{~s}^{-1}$ in the Keck and MMT data sets. The decrease in $J$-factor due to the lower velocity dispersion is expected to be $\Delta \log _{10} J \sim-0.18$ or $\log _{10} J \sim$ 18.63 , which agrees with the $J$-factor calculation. Compared to the other data sets, we estimate differences of $\Delta \log _{10} J \sim 0.43,0.31$ just due to the changes in these inputs in Bonnivard et al. (2015) and Geringer-Sameth et al. (2015), respectively. Furthermore, we note that our structural parameters are derived from the spectroscopic

\footnotetext{
${ }^{13}$ See Evans, Sanders \& Geringer-Sameth (2016) and Ullio \& Valli (2016) for similar discussion.

${ }^{14}$ This was a preliminary catalogue of MMT/Hectochelle data eventually published in Spencer et al. (2018).
}

sample alone and are more uncertain than those derived from photometric catalogues due to the lower number of stars, improving the constraints on the metal-poor stellar distribution is a straightforward manner to improve the $J$-factor measurement. Other modelling assumptions such as the dark matter halo, velocity anisotropy, or stellar distribution can account for the remaining differences.

The $J$-factors derived from the metal-poor component are larger compared to the sample as a whole (RGB data set). Although the metal-poor component is more extended than UMi as a whole, the larger $\sigma_{v}$ implies an overall denser dark matter halo. The spherical Jeans modelling is more applicable to the more spherical metalpoor population than UMi as a whole because of the ellipticity. This suggests that future work with axisymmetric mass models will be particularly beneficial for understanding UMi (e.g. Zhu et al. 2016; Klop et al. 2017; Hayashi et al. 2018). Of the classical dSphs, UMi has one of the largest $J$-factors, our results suggest that it continues to be an excellent target, however, its kinematics are more complex than previously thought.

\section{CONCLUSION}

We have presented line-of-sight velocities and stellar metallicities from the largest spectroscopic data set of the classical dSph UMi. Our Keck/DEIMOS observations include 1630 measurements of the line-of-sight velocity and 1389 metallicity measurements of 1462 unique stars. Through a dSph and MW foreground mixture model, we utilized a combination of velocity, metallicity, position, and proper motion to identify 892 UMi members, doubling the number of known spectroscopic members.

We have discussed a methodology for disentangling chemodynamical populations in $\mathrm{dSph}$ galaxies building upon previous work by extending the analysis to axisymmetry and utilizing Bayesian evidence to compare models with and without multiple populations. We have uncovered two chemodynamic stellar populations in UMi at high statistical significance $(\ln B=33.47)$. The first population is more metal-rich $(\overline{[\mathrm{Fe} / \mathrm{H}]} \approx-2.05)$, kinematically cold $\left(\sigma_{v} \approx\right.$ $\left.4.9 \mathrm{~km} \mathrm{~s}^{-1}\right)$, and centrally concentrated $\left(R_{\mathrm{h}} \approx 220 \mathrm{pc}\right)$ compared to the second more metal-poor $(\overline{[\mathrm{Fe} / \mathrm{H}]} \approx-2.29)$, kinematically hot $\left(\sigma_{v} \approx 11.5 \mathrm{~km} \mathrm{~s}^{-1}\right)$, and extended population $\left(R_{\mathrm{h}} \approx 370 \mathrm{pc}\right)$. The two populations in UMi follow the same chemodynamical ordering observed in other dSphs (e.g. Sculptor and Fornax; Battaglia et al. 2008; Walker \& Peñarrubia 2011).

We applied the same methodology to a smaller independent MMT/Hectochelle spectroscopic data set (Spencer et al. 2018). Our analysis of this sample confirmed our discovery of two chemodynamic populations in UMi ( $\ln B=17.94)$. The properties of the chemodynamical populations with the MMT data set are overall similar to the Keck/DEIMOS results, although we find that the metal-rich velocity dispersion and the metal-poor spatial scale are larger compared to the Keck data set.

In both data sets, the UMi metal-rich population is significantly more elongated $(\epsilon=0.75 \pm 0.03)$ than the almost spherical metalpoor population. The large difference in ellipticity may be a hint of different formation mechanisms. The velocity dispersion of the metal-poor population decreases from $\approx 13 \mathrm{~km} \mathrm{~s}^{-1}$ at the centre of $\mathrm{UMi}$ to $\approx 10 \mathrm{~km} \mathrm{~s}^{-1}$ at the edge of our sample. We searched for and found some hints of prolate rotation in UMi in the MMT data set. However, in the Keck data set we found no evidence for rotation. We further explored whether a three-population chemodynamic model better explains the UMi data. There is some evidence of three populations in the Keck data set, however, the 'new' population is a split of the original metal-poor component suggesting that the metal- 
Table 6. The $J$ - and $D$-factors for UMi integrated over several solid angles with the RGB sample and the metal-poor sample for the Keck/DEIMOS and MMT/Hectochelle data sets. The $J$ - and $D$-factors have units of $\mathrm{GeV}^{2} \mathrm{~cm}^{-5}$ and $\mathrm{GeV} \mathrm{cm}^{-2}$, respectively. For reference, $1 \mathrm{M}_{\odot}^{2} \mathrm{kpc}^{-5}=4.45 \times 10^{6} \mathrm{GeV}^{2} \mathrm{~cm}^{-5}$ and $1 \mathrm{M}_{\odot} \mathrm{kpc}^{-2}=1.17 \times 10^{14} \mathrm{GeV} \mathrm{cm}^{-2}$. We list the $1 \sigma$ and $2 \sigma$ error bars.

\begin{tabular}{|c|c|c|c|c|c|c|c|}
\hline Model & $\log _{10} J(0.1)$ & $\log _{10} J(0.2)$ & $\log _{10} J(0.5)$ & $\log _{10} J(1.0)$ & $\log _{10} D(0.1)$ & $\log _{10} D(0.2)$ & $\log _{10} D(0.5)$ \\
\hline Keck - MP & $18.80_{-0.33(0.68)}^{+0.30(0.58)}$ & $18.98_{-0.21(0.43)}^{+0.21(0.45)}$ & $19.08_{-0.13(0.23)}^{+0.16(0.37)}$ & $19.11_{-0.12(0.23)}^{+0.16(0.36)}$ & $17.51_{-0.06(0.13)}^{+0.05(0.10)}$ & $17.93_{-0.05(0.09)}^{+0.06(0.14)}$ & $17.93_{-0.18(0.32)}^{+0.19(0.37)}$ \\
\hline Keck - RGB & $18.52_{-0.41(0.72)}^{+0.31(0.51)}$ & $18.64_{-0.22(0.38)}^{+0.24(0.42)}$ & $18.73_{-0.11(0.18)}^{+0.17(0.34)}$ & $18.78_{-0.12(0.20)}^{+0.17(0.33)}$ & $17.31_{-0.03(0.07)}^{+0.03(0.08)}$ & $17.70_{-0.05(0.09)}^{+0.11(0.22)}$ & $17.64_{-0.23(0.34)}^{+0.28(0.53)}$ \\
\hline MMT - MP & $18.01_{-0.59(1.15)}^{+0.55(1.04)}$ & $18.34_{-0.46(0.94)}^{+0.43(0.83)}$ & $18.61_{-0.31(0.62)}^{+0.31(0.63)}$ & $18.71_{-0.25(0.48)}^{+0.26(0.56)}$ & $17.27_{-0.16(0.32)}^{+0.16(0.30)}$ & $17.77_{-0.13(0.26)}^{+0.12(0.24)}$ & $17.94_{-0.20(0.47)}^{+0.14(0.27)}$ \\
\hline MMT - RGB & $18.16_{-0.22(0.48)}^{+0.28(0.59)}$ & $18.37_{-0.14(0.32)}^{+0.18(0.42)}$ & $18.47_{-0.09(0.18)}^{+0.13(0.35)}$ & $18.49_{-0.09(0.17)}^{+0.13(0.34)}$ & $17.21_{-0.05(0.11)}^{+0.05(0.09)}$ & $17.64_{-0.03(0.06)}^{+0.03(0.07)}$ & $17.70_{-0.11(0.22)}^{+0.12(0.27)}$ \\
\hline
\end{tabular}

poor component may have a non-Gaussian metallicity distribution. In contrast, the three-population results with the MMT data do not have consistent results with different priors and disagree with the Keck results. Additional extended data (especially along the minor axis) will be key for determining if UMi exhibits prolate rotation or if UMi is split into additional chemodynamic populations. Future spectroscopic observations can utilize Gaia astrometry to remove a large portion of the MW foreground and increase the yield at large radii.

We explored a simple mass-slope estimator, $\Gamma$ (Walker \& Peñarrubia 2011), to probe the dark matter distribution. The $\Gamma$ distribution disfavours a 'cuspy' halo with the Keck data. However, the naive interpretation of very large $\Gamma$ values implies that the density increases with radius. In contrast, the MMT data favour a 'cuspy' slope but is still consistent with 'cored' haloes. The flattened metal-rich population, potential stellar rotation, and the non-constant metal-poor velocity dispersion invalidate several of the assumptions this estimator is based on. More detailed modelling is required to robustly determine the dark matter density distribution in UMi. In addition, it would be interesting to combine line-of-sight velocities with proper motion measurements obtained from Gaia to better constrain the inner slope of the dark matter mass profile mass slope (Strigari, Bullock \& Kaplinghat 2007b; Massari et al. 2018, 2020).

We have calculated the astrophysical components ( $J$ - and $D$-factor) for dark matter annihilation or decay based on the inferred dark matter densities from spherical Jeans fits. In particular, we modelled the rounder metal-poor component as it is less prone to modelling systematics due to flattening. We derived $\log _{10}\left(J(0.5) / \mathrm{Gev}^{-2} \mathrm{~cm}^{-5}\right) \approx 19.1$ and $\log _{10}\left(J(0.5) / \mathrm{Gev}^{-2} \mathrm{~cm}^{-5}\right) \approx 18.6$ with the Keck and MMT data set, respectively. Thus, UMi is an excellent target for searches for dark matter annihilation or decay.

The presence of a highly flattened metal-rich population in UMi is unexpected and deserves a closer look. If the hints for prolate rotation are confirmed, then that will add to the puzzle of the formation and evolution of UMi. The methods we have developed for the analysis of UMi can be applied to other classical dSphs to characterize their multiple populations and constrain their dark matter profiles more robustly.

\section{ACKNOWLEDGEMENTS}

We thank Matt Walker for sharing an earlier catalogue of the UMi MMT/Hectochelle data. We thank James Bullock, Mike Cooper, Sergey Koposov, Jen Marshall, Louie Strigari, and Matt Walker for helpful comments and discussion. We also thank the referee for their helpful comments.

ABP was supported by a GAANN fellowship at UCI. ABP acknowledges generous support from the George P. and Cynthia
Woods Institute for Fundamental Physics and Astronomy at Texas A\&M University. ABP was supported by NSF grant AST-1813881. EK gratefully acknowledges support from a Cottrell Scholar award administered by the Research Corporation for Science Advancement and funding from generous donors to the California Institute of Technology. RRM acknowledges partial support from project BASAL AFB-170002 and FONDECYT project $\mathrm{N}^{\circ} 1170364$. SGD was supported in part by the NSF grants AST-1413600, AST1518308, and AST-1749235.

The authors wish to recognize and acknowledge the very significant cultural role and reverence that the summit of Mauna Kea has always had within the indigenous Hawaiian community. We are most fortunate to have the opportunity to conduct observations from this mountain.

Funding for SDSS-III has been provided by the Alfred P. Sloan Foundation, the Participating Institutions, the National Science Foundation, and the US Department of Energy Office of Science. The SDSS-III web site is http://www.sdss3.org/.

SDSS-III is managed by the Astrophysical Research Consortium for the Participating Institutions of the SDSS-III Collaboration including the University of Arizona, the Brazilian Participation Group, Brookhaven National Laboratory, Carnegie Mellon University, University of Florida, the French Participation Group, the German Participation Group, Harvard University, the Instituto de Astrofisica de Canarias, the Michigan State/Notre Dame/JINA Participation Group, Johns Hopkins University, Lawrence Berkeley National Laboratory, Max Planck Institute for Astrophysics, Max Planck Institute for Extraterrestrial Physics, New Mexico State University, New York University, Ohio State University, Pennsylvania State University, University of Portsmouth, Princeton University, the Spanish Participation Group, University of Tokyo, University of Utah, Vanderbilt University, University of Virginia, University of Washington, and Yale University.

This work has made use of data from the European Space Agency (ESA) mission Gaia (https://www.cosmos.esa.int/gaia), processed by the Gaia Data Processing and Analysis Consortium (DPAC, ht tps://www.cosmos.esa.int/web/gaia/dpac/consortium). Funding for the DPAC has been provided by national institutions, in particular the institutions participating in the Gaia Multilateral Agreement.

This research has made use of NASA's Astrophysics Data System Bibliographic Services.

Databases and software: Besançon model ${ }^{15}$ (Robin et al. 2003). PYTHON packages: ASTROPY ${ }^{16}$ (Astropy Collaboration 2013), NUMPY (Walt, Colbert \& Varoquaux 2011), IPYTHON (Pérez \& Granger 2007), SCIPY (Jones et al. 2001), MATPLOTLIB (Hunter 2007), CORNER.PY (Foreman-Mackey 2016), and EMCEE (ForemanMackey et al. 2013).

\footnotetext{
${ }^{15} \mathrm{http}: / /$ model.obs-besancon.fr/

${ }^{16} \mathrm{http} / / /$ www.astropy.org
} 
The data presented in this work were obtained at the W.M. Keck Observatory, which is operated as a scientific partnership among the California Institute of Technology, the University of California and the National Aeronautics and Space Administration. The observatory was made possible by the generous financial support of the W.M. Keck Foundation.

\section{REFERENCES}

Abazajian K. N., 2014, Phys. Rev. Lett., 112, 161303

Abazajian K. N. et al., 2009, ApJS, 182, 543

Ackermann M. et al., 2014, Phys. Rev. D, 89, 042001

Adams J. J. et al., 2014, ApJ, 789, 63

Agnello A., Evans N. W., 2012, ApJ, 754, L39

Ahn C. P. et al., 2012, ApJS, 203, 21

Aihara H. et al., 2011a, ApJS, 193, 29

Aihara H. et al., 2011b, ApJS, 195, 26

Amorisco N. C., Evans N. W., 2012a, MNRAS, 419, 184

Amorisco N. C., Evans N. W., 2012b, ApJ, 756, L2

Amorisco N. C., Agnello A., Evans N. W., 2013, MNRAS, 429, L89

Amorisco N. C., Evans N. W., van de Ven G., 2014, Nature, 507, 335

Astropy Collaboration, 2013, A\&A, 558, A33

Baes M., van Hese E., 2007, A\&A, 471, 419

Baltz E. A., Briot C., Salati P., Taillet R., Silk J., 2000, Phys. Rev. D, 61, 023514

Battaglia G. et al., 2006, A\&A, 459, 423

Battaglia G., Helmi A., Tolstoy E., Irwin M., Hill V., Jablonka P., 2008, ApJ, 681, L13

Battaglia G., Tolstoy E., Helmi A., Irwin M., Parisi P., Hill V., Jablonka P., 2011, MNRAS, 411, 1013

Bellazzini M., Ferraro F. R., Origlia L., Pancino E., Monaco L., Oliva E., 2002, AJ, 124, 3222

Bergström L., Hooper D., 2006, Phys. Rev. D, 73, 063510

Binney J., Tremaine S., 2008, Galactic Dynamics, 2nd edn. Princeton Univ. Press, Princeton, NJ

Bonnivard V. et al., 2015, MNRAS, 453, 849

Brooks A. M., Kuhlen M., Zolotov A., Hooper D., 2013, ApJ, 765, 22

Campbell D. J. R. et al., 2017, MNRAS, 469, 2335

Chambers K. C. et al., 2016, preprint (arXiv:1612.05560)

Cicuéndez L., Battaglia G., 2018, MNRAS, 480, 251

Clementini G. et al., 2019, A\&A, 622, A60

Cohen J. G., 1978, ApJ, 221, 788

Colafrancesco S., Profumo S., Ullio P., 2007, Phys. Rev. D, 75, 023513

Collins M. L. M. et al., 2013, ApJ, 768, 172

Cooper M. C., Newman J. A., Davis M., Finkbeiner D. P., Gerke B. F., 2012, Astrophysics Source Code Library, record ascl:1203.003

de Blok W. J. G., Bosma A., 2002, A\&A, 385, 816

de Blok W. J. G., Walter F., Brinks E., Trachternach C., Oh S.-H., Kennicutt R. C., Jr, 2008, AJ, 136, 2648

de Boer T. J. L., Tolstoy E., Lemasle B., Saha A., Olszewski E. W., Mateo M., Irwin M. J., Battaglia G., 2014, A\&A, 572, A10

del Pino A., Aparicio A., Hidalgo S. L., 2015, MNRAS, 454, 3996

del Pino A., Aparicio A., Hidalgo S. L., Łokas E. L., 2017a, MNRAS, 465, 3708

del Pino A., Łokas E. L., Hidalgo S. L., Fouquet S., 2017b, MNRAS, 469, 4999

Demarque P., Woo J.-H., Kim Y.-C., Yi S. K., 2004, ApJS, 155, 667

Dotter A., Chaboyer B., Jevremović D., Baron E., Ferguson J. W., Sarajedini A., Anderson J., 2007, AJ, 134, 376

Dotter A., Chaboyer B., Jevremović D., Kostov V., Baron E., Ferguson J. W., 2008, ApJS, 178, 89

Eadie G. M., Harris W. E., 2016, ApJ, 829, 108

Ebrová I., Łokas E. L., 2017, ApJ, 850, 144

Einasto J., 1965, Trudy Astrofizicheskogo Inst. Alma-Ata, 5, 87

Errani R., Peñarrubia J., Walker M. G., 2018, MNRAS, 481, 5073

Evans N. W., Ferrer F., Sarkar S., 2004, Phys. Rev. D, 69, 123501

Evans N. W., Sanders J. L., Geringer-Sameth A., 2016, Phys. Rev. D, 93 , 103512
Faber S. M. et al., 2003, in Iye M., Moorwood A. F. M., eds, Proc. SPIE Vol. 4841, Instrument Design and Performance for Optical/Infrared GroundBased Telescopes. SPIE, Bellingham, p. 1657

Fabrizio M. et al., 2011, PASP, 123, 384

Fabrizio M. et al., 2016, ApJ, 830, 126

Feast M. W., Thackeray A. D., Wesselink A. J., 1961, MNRAS, 122, 433

Feroz F., Hobson M. P., 2008, MNRAS, 384, 449

Feroz F., Hobson M. P., Bridges M., 2009, MNRAS, 398, 1601

Fillingham S. P. et al., 2019, preprint (arXiv:1906.04180)

Foreman-Mackey D., 2016, J. Open Source Software, 1, 24

Foreman-Mackey D., Hogg D. W., Lang D., Goodman J., 2013, PASP, 125, 306

Fouquet S., Łokas E. L., del Pino A., Ebrová I., 2017, MNRAS, 464, 2717

Fritz T. K., Battaglia G., Pawlowski M. S., Kallivayalil N., van der Marel R., Sohn S. T., Brook C., Besla G., 2018, A\&A, 619, A103

Gaia Collaboration, 2018a, A\&A, 616, A1

Gaia Collaboration, 2018b, A\&A, 616, A12

Genina A. et al., 2018, MNRAS, 474, 1398

Genina A., Frenk C. S., Benítez-Llambay A. R., Cole S., Navarro J. F., Oman K. A., Fattahi A., 2019, MNRAS, 488, 2312

Geringer-Sameth A., Koushiappas S. M., Walker M., 2015, ApJ, 801, 74

Gibbons S. L. J., Belokurov V., Evans N. W., 2017, MNRAS, 464, 794

Gilbert K. M. et al., 2018, ApJ, 852, 128

Girardi L., Grebel E. K., Odenkirchen M., Chiosi C., 2004, A\&A, 422, 205

González-Samaniego A., Bullock J. S., Boylan-Kolchin M., Fitts A., Elbert O. D., Hopkins P. F., Kereš D., Faucher-Giguère C.-A., 2017, MNRAS, 472,4786

Governato F. et al., 2010, Nature, 463, 203

Governato F. et al., 2012, MNRAS, 422, 1231

Harbeck D. et al., 2001, AJ, 122, 3092

Hayashi K., Fabrizio M., Łokas E. L., Bono G., Monelli M., Dall'Ora M., Stetson P. B., 2018, MNRAS, 481, 250

Hendricks B., Koch A., Walker M., Johnson C. I., Peñarrubia J., Gilmore G., 2014, A\&A, 572, A82

Ho N. et al., 2012, ApJ, 758, 124

Horigome S.-i., Hayashi K., Ibe M., Ishigaki M. N., Matsumoto S., Sugai H., 2020, preprint (arXiv:2002.04866)

Horiuchi S., Humphrey P. J., Oñorbe J., Abazajian K. N., Kaplinghat M., Garrison-Kimmel S., 2014, Phys. Rev. D, 89, 025017

Horne K., 1986, PASP, 98, 609

Hunter J. D., 2007, Comput. Sci. Eng., 9, 90

Hurley-Keller D., Mateo M., Nemec J., 1998, AJ, 115, 1840

Ibata R., Chapman S., Irwin M., Lewis G., Martin N., 2006, MNRAS, 373, L70

Jones E. et al., 2001, SciPy: Open Source Scientific Tools for Python. http://www.scipy.org/

Kacharov N. et al., 2017, MNRAS, 466, 2006

Kamada A., Kaplinghat M., Pace A. B., Yu H.-B., 2017, Phys. Rev. Lett., 119,111102

Kaplinghat M., Strigari L. E., 2008, ApJ, 682, L93

Kaplinghat M., Keeley R. E., Linden T., Yu H.-B., 2014, Phys. Rev. Lett., 113, 021302

Kaplinghat M., Tulin S., Yu H.-B., 2016, Phys. Rev. Lett., 116, 041302

Kim H.-S., Han S.-I., Joo S.-J., Jeong H., Yoon S.-J., 2019, ApJ, 870, L8

Kirby E. N., Guhathakurta P., Sneden C., 2008, ApJ, 682, 1217

Kirby E. N. et al., 2010, ApJS, 191, 352

Kirby E. N., Lanfranchi G. A., Simon J. D., Cohen J. G., Guhathakurta P., 2011, ApJ, 727, 78

Kirby E. N., Simon J. D., Cohen J. G., 2015, ApJ, 810, 56

Kirby E. N., Xie J. L., Guo R., Kovalev M., Bergemann M., 2018, ApJS, 237, 18

Kleyna J. T., Wilkinson M. I., Gilmore G., Evans N. W., 2003, ApJ, 588, L21

Kleyna J. T., Wilkinson M. I., Evans N. W., Gilmore G., 2004, MNRAS, 354, L66

Klop N., Zandanel F., Hayashi K., Ando S., 2017, Phys. Rev. D, 95, 123012

Koposov S. E. et al., 2011, ApJ, 736, 146

Kordopatis G., Amorisco N. C., Evans N. W., Gilmore G., Koposov S. E., 2016, MNRAS, 457, 1299 
Kowalczyk K., Łokas E. L., Kazantzidis S., Mayer L., 2013, MNRAS, 431, 2796

Kuzio de Naray R., McGaugh S. S., de Blok W. J. G., Bosma A., 2006, ApJS, 165, 461

Kuzio de Naray R., McGaugh S. S., de Blok W. J. G., 2008, ApJ, 676, 920

Laporte C. F. P., Walker M. G., Peñarrubia J., 2013, MNRAS, 433, L54

Lokas E. L., Ebrova I., Del Pino A., Semczuk M., 2014, MNRAS, 445, L6

Lora V., Grebel E. K., Schmeja S., Koch A., 2019, ApJ, 878, 152

Lovell M. R., Frenk C. S., Eke V. R., Jenkins A., Gao L., Theuns T., 2014, MNRAS, 439, 300

Lyskova N., Thomas J., Churazov E., Tremaine S., Naab T., 2015, MNRAS, 450,3442

McConnachie A. W., 2012, AJ, 144, 4

McConnachie A. W., Peñarrubia J., Navarro J. F., 2007, MNRAS, 380, L75

Majewski S. R. et al., 2013, ApJ, 777, L13

Martin N. F., Ibata R. A., Chapman S. C., Irwin M., Lewis G. F., 2007, MNRAS, 380, 281

Martinez G. D., Minor Q. E., Bullock J., Kaplinghat M., Simon J. D., Geha M., 2011, ApJ, 738, 55

Massari D., Breddels M. A., Helmi A., Posti L., Brown A. G. A., Tolstoy E., 2018, Nat. Astron., 2, 156

Massari D., Helmi A., Mucciarelli A., Sales L. V., Spina L., Tolstoy E., 2020, A\&A, 633, A36

Muñoz R. R. et al., 2005, ApJ, 631, L137

Muñoz R. R., Carlin J. L., Frinchaboy P. M., Nidever D. L., Majewski S. R., Patterson R. J., 2006, ApJ, 650, L51

Muñoz R. R., Côté P., Santana F. A., Geha M., Simon J. D., Oyarzún G. A., Stetson P. B., Djorgovski S. G., 2018a, ApJ, 860, 65

Muñoz R. R., Côté P., Santana F. A., Geha M., Simon J. D., Oyarzún G. A., Stetson P. B., Djorgovski S. G., 2018b, ApJ, 860, 66

Navarro J. F., Eke V. R., Frenk C. S., 1996a, MNRAS, 283, L72

Navarro J. F., Frenk C. S., White S. D. M., 1996b, ApJ, 462, 563

Navarro J. F., Frenk C. S., White S. D. M., 1997, ApJ, 490, 493

Navarro J. F. et al., 2004, MNRAS, 349, 1039

Newman J. A. et al., 2013, ApJS, 208, 5

Oh S.-H., de Blok W. J. G., Brinks E., Walter F., Kennicutt R. C., Jr, 2011, AJ, 141, 193

Oh S.-H. et al., 2015, AJ, 149, 180

Oñorbe J., Boylan-Kolchin M., Bullock J. S., Hopkins P. F., Kereš D., Faucher-Giguère C.-A., Quataert E., Murray N., 2015, MNRAS, 454, 2092

Pace A. B., Li T. S., 2019, ApJ, 875, 77

Pace A. B., Strigari L. E., 2019, MNRAS, 482, 3480

Pace A. B., Martinez G. D., Kaplinghat M., Muñoz R. R., 2014, MNRAS, 442,1718

Palma C., Majewski S. R., Siegel M. H., Patterson R. J., Ostheimer J. C., Link R., 2003, AJ, 125, 1352

Pasetto S., Grebel E. K., Berczik P., Chiosi C., Spurzem R., 2011, A\&A, 525, A99

Peñarrubia J., Pontzen A., Walker M. G., Koposov S. E., 2012, ApJ, 759, L42

Pérez F., Granger B. E., 2007, Comput. Sci. Eng., 9, 21

Peter A. H. G., Rocha M., Bullock J. S., Kaplinghat M., 2013, MNRAS, 430,105

Plummer H. C., 1911, MNRAS, 71, 460

Pontzen A., Governato F., 2012, MNRAS, 421, 3464

Read J. I., Steger P., 2017, MNRAS, 471, 4541

Relatores N. C. et al., 2019, ApJ, 887, 94

Revaz Y., Jablonka P., 2018, A\&A, 616, A96

Revaz Y. et al., 2009, A\&A, 501, 189

Robin A. C., Reylé C., Derrière S., Picaud S., 2003, A\&A, 409, 523

Rocha M., Peter A. H. G., Bullock J. S., Kaplinghat M., Garrison-Kimmel S., Oñorbe J., Moustakas L. A., 2013, MNRAS, 430, 81

Salvadori S., Ferrara A., Schneider R., 2008, MNRAS, 386, 348

Sesar B. et al., 2017, AJ, 153, 204

Simon J. D., 2019, ARA\&A, 57, 375

Simon J. D., Geha M., 2007, ApJ, 670, 313
Simon J. D., Bolatto A. D., Leroy A., Blitz L., Gates E. L., 2005, ApJ, 621 , 757

Skilling J., 2004, in Fischer R., Preuss R., von Toussaint U., eds, AIP Conf. Proc. Vol. 735, Bayesian Inference and Maximum Entropy Methods in Science and Engineering. Am. Inst. Phys., New York, p. 395

Sohn S. T. et al., 2007, ApJ, 663, 960

Spencer M. E., Mateo M., Walker M. G., Olszewski E. W., McConnachie A. W., Kirby E. N., Koch A., 2017, AJ, 153, 254

Spencer M. E., Mateo M., Olszewski E. W., Walker M. G., McConnachie A. W., Kirby E. N., 2018, AJ, 156, 257

Spinrad H., Taylor B. J., 1971, ApJS, 22, 445

Springel V. et al., 2008, MNRAS, 391, 1685

Strigari L. E., Koushiappas S. M., Bullock J. S., Kaplinghat M., 2007a, Phys. Rev. D, 75, 083526

Strigari L. E., Bullock J. S., Kaplinghat M., 2007b, ApJ, 657, L1

Strigari L. E., Koushiappas S. M., Bullock J. S., Kaplinghat M., Simon J. D., Geha M., Willman B., 2008, ApJ, 678, 614

Strigari L. E., Frenk C. S., White S. D. M., 2017, ApJ, 838, 123

Taibi S. et al., 2018, A\&A, 618, A122

Taibi S., Battaglia G., Rejkuba M., Leaman R., Kacharov N., Iorio G., Jablonka P., Zoccali M., 2020, A\&A, 635, A152

Tollerud E. J. et al., 2012, ApJ, 752, 45

Tolstoy E. et al., 2004, ApJ, 617, L119

Tonry J., Davis M., 1979, AJ, 84, 1511

Trotta R., 2008, Contemporary Phys., 49, 71

Tyler C., 2002, Phys. Rev. D, 66, 023509

Ullio P., Valli M., 2016, J. Cosmol. Astropart. Phys., 07, 025

Ural U. et al., 2010, MNRAS, 402, 1357

van der Marel R. P., Alves D. R., Hardy E., Suntzeff N. B., 2002, AJ, 124, 2639

Walker M. G., Peñarrubia J., 2011, ApJ, 742, 20

Walker M. G., Mateo M., Olszewski E. W., Pal J. K., Sen B., Woodroofe M., 2006, ApJ, 642, L41

Walker M. G., Mateo M., Olszewski E. W., Gnedin O. Y., Wang X., Sen B., Woodroofe M., 2007, ApJ, 667, L53

Walker M. G., Mateo M., Olszewski E. W., 2008, ApJ, 688, L75

Walker M. G., Mateo M., Olszewski E. W., Peñarrubia J., Wyn Evans N., Gilmore G., 2009, ApJ, 704, 1274

Walker M. G., Combet C., Hinton J. A., Maurin D., Wilkinson M. I., 2011, ApJ, 733, L46

Walt S. v. d., Colbert S. C., Varoquaux G., 2011, Comput. Sci. Eng., 13, 22

Wang X., Woodroofe M., Walker M. G., Mateo M., Olszewski E., 2005, ApJ, 626, 145

Wang M.-Y., Peter A. H. G., Strigari L. E., Zentner A. R., Arant B., GarrisonKimmel S., Rocha M., 2014, MNRAS, 445, 614

Weisz D. R., Dolphin A. E., Skillman E. D., Holtzman J., Gilbert K. M., Dalcanton J. J., Williams B. F., 2014, ApJ, 789, 147

Wheeler C. et al., 2017, MNRAS, 465, 2420

Wilkinson M. I., Kleyna J. T., Evans N. W., Gilmore G. F., Irwin M. J., Grebel E. K., 2004, ApJ, 611, L21

Wolf J., Martinez G. D., Bullock J. S., Kaplinghat M., Geha M., Muñoz R. R., Simon J. D., Avedo F. F., 2010, MNRAS, 406, 1220

Zhu L., van de Ven G., Watkins L. L., Posti L., 2016, MNRAS, 463, 1117

\section{SUPPORTING INFORMATION}

Supplementary data are available at MNRAS online.

\section{table_keck_v2}

Please note: Oxford University Press is not responsible for the content or functionality of any supporting materials supplied by the authors. Any queries (other than missing material) should be directed to the corresponding author for the article.

This paper has been typeset from a $\mathrm{T}_{\mathrm{E}} \mathrm{X} / \mathrm{L} \mathrm{T}_{\mathrm{E}} \mathrm{X}$ file prepared by the author. 